\title{
INSENSITIZING CONTROLS WITH TWO VANISHING COMPONENTS FOR THE THREE-DIMENSIONAL BOUSSINESQ SYSTEM
}

\author{
N. Carreño ${ }^{1}$, S. Guerrero ${ }^{1}$ and M. Gueye ${ }^{1}$
}

\begin{abstract}
In this paper we prove the existence of insensitizing controls for a viscous newtonian fluid wherein thermic effects are taken into acount, the so called Boussinesq system. The proof relies on a standard approach introduced by Fursikov and Imanuvilov for the Navier-Stokes system which consists in solving a constrained extremal problem, and then on an inverse mapping theorem to deal with the nonlinear system. Furthermore, we use the coupling with the heat equation to get rid of two components of the control in the fluid equations.
\end{abstract}

Mathematics Subject Classification. 34B15, 35Q30, 93C10, 93B05.

Received January 31, 2013. Revised January 1, 2014.

Published online October 17, 2014.

\section{INTRODUCTION}

Let $\Omega$ be a nonempty bounded connected open subset of $\mathbb{R}^{N}(N=2$ or 3$)$ of class $\mathcal{C}^{\infty}$. Let $T>0$ and let $\omega \subset \Omega$ be a (small) nonempty open subset which is the control set. We will use the notation $Q=\Omega \times(0, T)$ and $\Sigma=\partial \Omega \times(0, T)$. Let us also introduce another open set $\mathcal{O} \subset \Omega$ which is called the observatory or observation set.

Let us recall the definition of some usual spaces in the context of incompressible fluids:

$$
\mathcal{V}=\left\{y \in \mathcal{C}_{0}^{\infty}(\Omega)^{N}: \nabla \cdot y=0 \text { in } \Omega\right\} .
$$

We denote by $H$ the closure of the space $\mathcal{V}$ in $L^{2}(\Omega)$ and by $V$ its closure in $H_{0}^{1}(\Omega)$.

We introduce the following Boussinesq control system with incomplete data:

$$
\begin{cases}y_{t}-\Delta y+(y \cdot \nabla) y+\nabla p=f+v \mathbb{1}_{\omega}+\theta e_{N}, \nabla \cdot y=0 & \text { in } \quad Q, \\ \theta_{t}-\Delta \theta+y \cdot \nabla \theta=f_{0}+v_{0} \mathbb{1}_{\omega} & \text { in } \quad Q, \\ y=0, \theta=0 & \text { on } \Sigma, \\ y(0)=y^{0}+\tau \widehat{y}_{0}, \theta(0)=\theta^{0}+\tau \widehat{\theta}_{0} & \text { in } \Omega .\end{cases}
$$

Here,

$$
e_{N}=\left\{\begin{array}{lll}
(0,1) & \text { if } & N=2, \\
(0,0,1) & \text { if } & N=3,
\end{array}\right.
$$

Keywords and phrases. Navier-Stokes system, Boussinesq system, null controllability, Carleman inequalities, insensitizing controls.

1 Université Pierre et Marie Curie, UMR 7598, Laboratoire Jacques-Louis Lions, 75005 Paris, France.

ncarreno@ann.jussieu.fr; guerrero@ann.jussieu.fr; gueye@ann.jussieu.fr 
stands for the gravity vector field, $y=y(x, t)$ represents the velocity of the particules of the fluid, $\theta=\theta(x, t)$ their temperature, $\left(v_{0}, v\right)=\left(v_{0}, v_{1}, \ldots, v_{N}\right)$ stands for the control which acts over the set $\omega,\left(f, f_{0}\right) \in L^{2}(Q)^{N+1}$ is a given externally applied force and the initial state $(y(0), \theta(0))$ is partially unknown in the following sense:

- $y^{0} \in H$ and $\theta^{0} \in L^{2}(\Omega)$ are known,

- $\widehat{y}_{0} \in H$ and $\widehat{\theta}_{0} \in L^{2}(\Omega)$ are unknown with $\left\|\widehat{y}_{0}\right\|_{L^{2}(\Omega)^{N}}=\left\|\widehat{\theta}_{0}\right\|_{L^{2}(\Omega)}=1$, and

- $\tau$ is a small unknown real number.

We observe the solution of system (1.1) via some functional $J_{\tau}(y, \theta)$, which is called the sentinel. Here, the sentinel is given by the square of the local $L^{2}$-norm of the state variables:

$$
J_{\tau}(y, \theta):=\frac{1}{2} \iint_{\mathcal{O} \times(0, T)}\left(|y|^{2}+|\theta|^{2}\right) \mathrm{d} x \mathrm{~d} t .
$$

The insensitizing control problem is to find $\left(v, v_{0}\right)$ such that the uncertainty in the initial data does not affect the measurement $J_{\tau}$, at least at the first order, i.e.,

$$
\left.\frac{\partial J_{\tau}(y, \theta)}{\partial \tau}\right|_{\tau=0}=0 \quad \forall\left(\widehat{y}_{0}, \widehat{\theta}_{0}\right) \in L^{2}(\Omega)^{N+1} \text { such that }\left\|\widehat{y}_{0}\right\|_{L^{2}(\Omega)^{N}}=\left\|\widehat{\theta}_{0}\right\|_{L^{2}(\Omega)}=1 .
$$

If (1.3) holds, we say that $v$ insensitizes the functional $J_{\tau}$. This kind of problem was first considered by Lions in $[19]$.

The first results of existence of insensitizing controls were obtained for the heat equation in [2, 26]. Both papers are concerned with a sentinel of the kind (1.2).

A very close subject is controllability with controls having some vanishing components, which may be an interesting field from the applications point of view. The first results were obtained in [9] for the local exact controllability to the trajectories of the Navier-Stokes and Boussinesq system when the closure of the control set $\omega$ intersects the boundary of $\Omega$. Later, this geometric assumption was removed for the Stokes system in [6], for the local null controllability of the Navier-Stokes system in [4] and in [3] for the Boussinesq system. Recently, the local null controllability of the three dimensional Navier-Stokes system with a control having two vanishing components has been obtained in [7].

As long as insensitivity results for fluids is concerned, the first result was obtained in [23], Section 2.3, where the author establishes the existence of $\varepsilon$-insensitizing controls of the form $\left(v_{1}, v_{2}, 0\right)$ for the $3 D$-Stokes system. Then, the existence of insensitizing controls for the Stokes system is proved in [11] and for the Navier-Stokes system in [12]. Finally, in [5], the existence of insensitizing controls for the Navier-Stokes system with one vanishing component was established. The present paper can be considered as a continuation of this last work. The main goal is to establish the existence of insensitizing controls for the Boussinesq system (1.1) having two vanishing components, that is, $v_{i_{0}} \equiv 0$ for any given $0<i_{0}<N$ and $v_{N} \equiv 0$. Notice that if $N=2$, this means $v \equiv 0$.

The particular form of the sentinel $J_{\tau}$ allows us to reformulate the insensitizing problem (1.1)-(1.3) as a controllability problem for a cascade system (for more details, see [2] or [18], for instance). In particular, condition (1.3) is equivalent to $(z(0), q(0))=0$ in $\Omega$, where $(z, q)$, together with $(w, r)$, solves the following coupled system:

$$
\begin{cases}w_{t}-\Delta w+(w \cdot \nabla) w+\nabla p_{0}=f+v \mathbb{1}_{\omega}+r e_{N}, \nabla \cdot w=0 & \text { in } \quad Q, \\ -z_{t}-\Delta z+\left(z \cdot \nabla^{t}\right) w-(w \cdot \nabla) z+q \nabla r+\nabla p_{1}=w \mathbb{1}_{\mathcal{O}}, \nabla \cdot z=0 & \text { in } \quad Q, \\ r_{t}-\Delta r+w \cdot \nabla r=f_{0}+v_{0} \mathbb{1}_{\omega} & \text { in } \quad Q, \\ -q_{t}-\Delta q-w \cdot \nabla q=z_{N}+r \mathbb{1}_{\mathcal{O}} & \text { in } \quad Q, \\ w=z=0, r=q=0 & \text { on } \Sigma, \\ w(0)=y^{0}, z(T)=0, r(0)=\theta^{0}, q(T)=0 & \text { in } \Omega .\end{cases}
$$


Here, $\left(w, p_{0}, r\right)$ is the solution of system (1.1) for $\tau=0$, the equation of $\left(z, p_{1}, q\right)$ corresponds to a formal adjoint of the equation satisfied by the derivative of $(y, \theta)$ with respect to $\tau$ at $\tau=0$ and we have denoted:

$$
\left(\left(z, \nabla^{t}\right) w\right)_{i}=\sum_{j=1}^{N} z_{j} \partial_{i} w_{j} \quad i=1, \ldots, N .
$$

Our main result is stated in the following theorem, which expresses local null-controllability of (1.4):

Theorem 1.1. Let $0<i_{0}<N$ and $m \geq 10$ be a real number. Assume that $\omega \cap \mathcal{O} \neq \emptyset, y^{0} \equiv 0$ and $\theta^{0} \equiv 0$. Then, there exist $\delta>0$ and $C>0$, depending on $\omega, \Omega, \mathcal{O}$ and $T$, such that for any $f \in L^{2}(Q)^{N}$ and any $f_{0} \in L^{2}(Q)$ satisfying $\left\|\mathrm{e}^{C / t^{m}}\left(f, f_{0}\right)\right\|_{L^{2}(Q)^{N+1}}<\delta$, there exists a control $\left(v, v_{0}\right) \in L^{2}(Q)^{N+1}$ with $v_{i_{0}} \equiv v_{N} \equiv 0$ such that the corresponding solution $(w, z, r, q)$ to (1.4) satisfies $z(0)=0$ and $q(0)=0$ in $\Omega$.

In particular, no control on the velocity equation is required when $N=2$.

Remark 1.2. The condition $\omega \cap \mathcal{O} \neq \emptyset$ has always been imposed as long as insensitizing controls are concerned. However, in [15], it has been proved that this is not a necessary condition for $\varepsilon$-insensitizing controls for some linear parabolic equations (see also [22]).

Remark 1.3. In [26], the author proved for the linear heat equation that we cannot expect insensitivity to hold for all initial data, except when the control acts everywhere in $\Omega$. Thus, we shall assume that $y^{0} \equiv 0$ and $\theta^{0} \equiv 0$.

To prove Theorem 1.1 we follow a standard approach introduced in [10] (see also [3, 5, 13]). We first deduce a null controllability result for the linear system:

$$
\begin{cases}w_{t}-\Delta w+\nabla p_{0}=f^{w}+v \mathbb{1}_{\omega}+r e_{N}, \nabla \cdot w=0 & \text { in } \quad Q, \\ -z_{t}-\Delta z+\nabla p_{1}=f^{z}+w \mathbb{1}_{\mathcal{O}}, \nabla \cdot z=0 & \text { in } \quad Q, \\ r_{t}-\Delta r=f^{r}+v_{0} \mathbb{1}_{\omega} & \text { in } Q, \\ -q_{t}-\Delta q=f^{q}+z_{N}+r \mathbb{1}_{\mathcal{O}} & \text { in } Q, \\ w=z=0, r=q=0 & \text { on } \Sigma, \\ w(0)=y^{0}, z(T)=0, r(0)=\theta^{0}, q(T)=0 & \text { in } \Omega,\end{cases}
$$

where $f^{w}, f^{z}, f^{r}$ and $f^{q}$ will be taken to decrease exponentially to zero at $t=0$.

The main tool to prove this controllability result for system (1.5), and the second main result of this paper, is a suitable Carleman estimate for the solutions of its adjoint system, namely,

$$
\begin{cases}-\varphi_{t}-\Delta \varphi+\nabla \pi_{\varphi}=g^{\varphi}+\psi \mathbb{1}_{\mathcal{O}}, \nabla \cdot \varphi=0 & \text { in } \quad Q, \\ \psi_{t}-\Delta \psi+\nabla \pi_{\psi}=g^{\psi}+\sigma e_{N}, \nabla \cdot \psi=0 & \text { in } \quad Q, \\ -\phi_{t}-\Delta \phi=g^{\phi}+\varphi_{N}+\sigma \mathbb{1}_{\mathcal{O}} & \text { in } \quad Q, \\ \sigma_{t}-\Delta \sigma=g^{\sigma} & \text { in } \quad Q, \\ \varphi=\psi=0, \phi=\sigma=0 & \text { on } \quad \Sigma, \\ \varphi(T)=0, \psi(0)=\psi^{0}, \phi(T)=0, \sigma(0)=\sigma^{0} & \text { in } \quad \Omega .\end{cases}
$$

Here, $\psi^{0} \in H, \sigma^{0} \in L^{2}(\Omega)$ and $g^{\varphi}, g^{\psi}, g^{\phi}$ and $g^{\sigma}$ will be taken with different regularity properties that will be detailed later on. In fact, this Carleman inequality is of the form

$$
\begin{aligned}
\iint_{Q} \widetilde{\rho}_{1}(t)\left(|\varphi|^{2}+|\psi|^{2}+|\phi|^{2}+|\sigma|^{2}\right) \mathrm{d} x \mathrm{~d} t \leq & C\left\|\widetilde{\rho}_{2}(t)\left(g^{\varphi}, g^{\psi}, g^{\phi}, g^{\sigma}\right)\right\|_{X}^{2} \\
& +(N-2) C \iiint_{\omega \times(0, T)} \widetilde{\rho}_{3}(t)\left|\varphi_{j_{0}}\right|^{2} \mathrm{~d} x \mathrm{~d} t+C \iint_{\omega \times(0, T)} \widetilde{\rho}_{4}(t)|\phi|^{2} \mathrm{~d} x \mathrm{~d} t,
\end{aligned}
$$


where $\widetilde{\rho}_{k}(t), k \in\{1,2,3,4\}$ are positive weight functions, $j_{0} \in\{1, \ldots, N-1\} \backslash\left\{i_{0}\right\}, C>0$ only depends on $\Omega$, $\omega, \mathcal{O}$ and $T$ and $X$ is a suitable Banach space. Observe that when $N=2$ the local term in $\varphi_{j_{0}}$ does not appear. This estimate is stated in Proposition 3.1.

Since the proof of (1.7) is quite technical, let us first give some insight about it. In particular, we are going to prove here the qualitative property corresponding to (1.7), that is to say

$$
(N-2) \varphi_{j_{0}} \equiv \phi \equiv 0 \quad \text { in } \omega \times(0, T) \Rightarrow \psi(T) \equiv \sigma(T) \equiv 0 \quad \text { in } \Omega .
$$

for the solutions of (1.6) with $\left(g^{\varphi}, g^{\psi}, g^{\phi}, g^{\sigma}\right)=(0,0,0,0)$.

Observe that, thanks to the backwards uniqueness for the heat and Stokes operator, $\psi(T) \equiv 0$ and $\sigma(T) \equiv$ 0 in $\Omega$ implies that $\varphi \equiv \psi \equiv 0$ and $\phi \equiv \sigma \equiv 0$ in $Q$.

Remark that (1.8) is equivalent to the approximate controllability of system (1.5) with $\left(f^{w}, f^{z}, f^{r}, f^{q}\right)=$ $(0,0,0,0)$

Proposition 1.4. Assume that $\omega \cap \mathcal{O} \neq \emptyset$ and $0<j_{0}<N$. Then, the unique continuation property (1.8) holds.

Remark 1.5. The proof of this unique continuation result follows the steps of the proof of (1.7). This is why we are going to divide its proof in several steps. In the lines below, we will make precise to which part of the proof of (1.7) corresponds each step.

Proof of Proposition 1.4. Without loss of generality, we can assume that $\psi^{0} \in \mathcal{V}$. Moreover, we can assume that $\sigma^{0} \in \mathcal{C}^{\infty}(\bar{\Omega})$. Now let us introduce the differential operators

$$
\mathcal{D}:=\partial_{1}^{2}+(N-2) \partial_{2}^{2}, \quad \mathcal{P}:=\Delta^{3}-\Delta \partial_{t}^{2} .
$$

We divide the proof in several steps:

Step 1. First we prove that the solutions of (1.6) satisfy

$$
\phi \equiv 0 \quad \text { in } \omega \times(0, T) \Rightarrow \mathcal{D} \sigma \equiv 0 \quad \text { in } Q .
$$

Straightforward computations, using in particular that $\Delta \pi_{\varphi}=0$ in $\mathcal{O} \times(0, T)$ and $\Delta \pi_{\psi}=\partial_{N} \sigma$ in $Q$, show that (for more details, see Sect. 3.3.1)

$$
-\mathcal{P} \phi_{t}-\Delta \mathcal{P} \phi=\mathcal{D} \sigma \quad \text { in } \mathcal{O} \times(0, T) .
$$

Then, we deduce that $\mathcal{D} \sigma=0$ in $(\omega \cap \mathcal{O}) \times(0, T)$. Furthermore, since the equation of $\sigma$ is homogeneous, $\mathcal{D} \sigma$ solves the heat equation

$$
(\mathcal{D} \sigma)_{t}-\Delta(\mathcal{D} \sigma)=0 \quad \text { in } Q .
$$

Then, from the parabolic unique continuation (see [24]) we deduce that $\mathcal{D} \sigma=0$ in $Q$.

Step 1 corresponds to estimate (3.29) in the proof of (1.7).

Step 2. Here, we observe that

$$
\mathcal{D} \sigma \equiv 0 \quad \text { in } Q \Rightarrow \sigma \equiv 0 \quad \text { in } Q .
$$

This fact is trivial from $\sigma=0$ on $\Sigma$.

Step 2 corresponds to estimate (3.15) in the proof of (1.7).

\section{Step 3.}

$$
\phi \equiv 0 \text { in } \omega \times(0, T), \sigma \equiv 0 \text { in } Q \Rightarrow \psi_{N} \equiv 0 \text { in } Q .
$$

From $\phi \equiv 0$ in $\omega \times(0, T)$ and $\sigma \equiv 0$ in $Q$ we get $\varphi_{N} \equiv 0$ in $\omega \times(0, T)$ (from the equation of $\left.\phi\right)$. Thus, from the equation satisfied by $\varphi_{N}$ we have

$$
\Delta \psi_{N}=-\left(\Delta \varphi_{N}\right)_{t}-\Delta\left(\Delta \varphi_{N}\right)=0 \text { in }(\omega \cap \mathcal{O}) \times(0, T) .
$$


Observe that $\Delta \psi_{N}$ satisfies the heat equation

$$
\left(\Delta \psi_{N}\right)_{t}-\Delta\left(\Delta \psi_{N}\right)=0 \text { in } Q .
$$

Making use of the parabolic unique continuation property we deduce that $\Delta \psi_{N} \equiv 0$ in $Q$. Then, the homogeneous Dirichlet boundary condition gives that $\psi_{N} \equiv 0$ in $Q$.

In dimension 2, this implies that $\psi \equiv 0$ in $Q$, since $\nabla \cdot \psi=0$ in $Q$ and $\left.\psi\right|_{\Sigma}=0$. This concludes the proof of Proposition 1.4 when $N=2$. Observe that we did not use that $\varphi_{j_{0}} \equiv 0$ in $\omega \times(0, T)$.

Step 4. The last step will be to prove that if $\sigma \equiv 0$ in $Q$, then

$$
\varphi_{j_{0}} \equiv \phi \equiv 0 \text { in } \omega \times(0, T) \Rightarrow \psi \equiv 0 \text { in } Q,
$$

for $0<j_{0}<N$. Using the fact that $\Delta \pi_{\varphi}=0$ in $\mathcal{O} \times(0, T)$, we find as before

$$
\Delta \psi_{j_{0}}=-\left(\Delta \varphi_{j_{0}}\right)_{t}-\Delta\left(\Delta \varphi_{j_{0}}\right)=0 \text { in }(\omega \cap \mathcal{O}) \times(0, T) .
$$

From $\sigma \equiv 0$ in $Q$, we have that $\Delta \pi_{\psi} \equiv 0$ in $Q$ and so

$$
\left(\Delta \psi_{j_{0}}\right)_{t}-\Delta\left(\Delta \psi_{j_{0}}\right)=0 \text { in } Q
$$

Thus, the parabolic unique continuation property applied to (1.10) gives $\Delta \psi_{j_{0}} \equiv 0$ in $Q$. Consequently, $\psi_{j_{0}} \equiv 0$ in $Q$, since $\psi_{j_{0} \mid \Sigma}=0$.

This, together with $\psi_{N} \equiv 0$ in $Q$ and combined with $\nabla \cdot \psi=0$ in $Q$ and $\psi_{\mid \Sigma}=0$ yield $\psi \equiv 0$ in $Q$.

Remark 1.6. Notice that after the first 3 steps, we have that $\psi_{3} \equiv 0$ in $Q$ and $\sigma \equiv 0$ in $Q$ using only $\phi \equiv$ 0 in $\omega \times(0, T)$. From previous results $[8,21]$, we can deduce that $\psi \equiv 0$ in $Q$ under some particular generic geometric conditions, that is, we are able to obtain the approximate controllability of system (1.1) with just a control in the heat equation.

The rest of the paper is organized as follows. In Section 2, we present some notation and the technical results we need. In Section 3, we prove the observability inequality (1.7). In Section 4, we prove a null controllability result for the linear system (1.5). Finally, by means of a inverse mapping theorem, we prove Theorem 1.1.

\section{TeChnical RESUlts And NOtATiOnS}

In this section we introduce some notation and all the technical results needed in this work.

\subsection{Some notations}

We denote by $X_{0}:=L^{2}(Q)$ and $Y_{0}:=L^{2}(0, T ; H)$. For $n$ a positive integer we define the spaces $X_{n}$ and $Y_{n}$ as follows:

$$
\begin{aligned}
X_{n} & :=L^{2}\left(0, T ; H^{2 n}(\Omega) \cap H_{0}^{1}(\Omega)\right) \cap H^{n}\left(0, T ; L^{2}(\Omega)\right), \\
Y_{n} & :=L^{2}\left(0, T ; H^{2 n}(\Omega)^{N} \cap V\right) \cap H^{n}\left(0, T ; L^{2}(\Omega)^{N}\right),
\end{aligned}
$$

endowed with the norms

$$
\|u\|_{X_{n}}^{2}:=\|u\|_{L^{2}\left(0, T ; H^{2 n}(\Omega)\right)}^{2}+\|u\|_{H^{n}\left(0, T ; L^{2}(\Omega)\right)}^{2}
$$

and

$$
\|u\|_{Y_{n}}^{2}:=\|u\|_{L^{2}\left(0, T ; H^{2 n}(\Omega)^{N}\right)}^{2}+\|u\|_{H^{n}\left(0, T ; L^{2}(\Omega)^{N}\right)}^{2},
$$

respectively. 
The following subspaces will be used only in Section 4. First, for every positive integer $n$, we set

$$
X_{n, 0}:=\left\{u \in X_{n}:\left[\mathcal{L}^{k} u\right]_{\mid \Sigma}=0,\left[\mathcal{L}^{k} u\right](0)=0, k=0, \ldots, n-1\right\},
$$

endowed with the equivalent norm (by Lem. 2.7),

$$
\|u\|_{X_{n, 0}}^{2}:=\left\|\mathcal{L}^{n} u\right\|_{L^{2}(Q)}^{2}
$$

where we have denoted by

$$
\mathcal{L}:=\partial_{t}-\Delta \quad \text { and } \quad \mathcal{L}^{*}:=-\partial_{t}-\Delta
$$

the heat operator and its adjoint, respectively.

Next, let

$$
Y_{1,0}:=\left\{u \in Y_{1}: u(0)=0\right\}
$$

and

$$
Y_{2,0}:=\left\{u \in Y_{1} \cap L^{2}\left(0, T ; H^{4}(\Omega)^{N}\right) \cap H^{2}\left(0, T ; L^{2}(\Omega)^{N}\right):\left(\mathcal{L}_{H} u\right)_{\mid \Sigma}=0,\left(\mathcal{L}_{H} u\right)(0)=0\right\}
$$

endowed with the equivalent norm (by Lem. 2.8 with $u_{0} \equiv 0$ )

$$
\|u\|_{Y_{n, 0}}^{2}:=\left\|\mathcal{L}_{H}^{n} u\right\|_{L^{2}(Q)^{N}}^{2}, \quad n=1,2 .
$$

Here, $\mathcal{L}_{H}:=\partial_{t}-\mathcal{P}_{L}(\Delta)$, where $\mathcal{P}_{L}$ denotes the Leray projector over the space $H$.

This equivalence between norms is used later to obtain Lemma (4.1).

\subsection{Carleman estimates}

In this subsection we present some Carleman estimates needed to prove estimate (1.7). This inequalities have been proved in previous papers and we give precise references about where to find each one of them. Before we can establish these estimates, let us introduce some classical weight functions. Let $\omega_{0}$ be a nonempty open subset of $\mathbb{R}^{N}$ such that $\omega_{0} \Subset \omega \cap \mathcal{O}$ and $\eta \in \mathcal{C}^{\infty}(\bar{\Omega})$ such that

$$
|\nabla \eta|>0 \quad \text { in } \quad \overline{\Omega \backslash \omega_{0}}, \eta>0 \quad \text { in } \quad \Omega \text { and } \eta \equiv 0 \quad \text { on } \quad \partial \Omega .
$$

The existence of such a function $\eta$ is given in [10]. Let also $\ell \in C^{\infty}([0, T])$ be a positive function in $(0, T)$ satisfying

$$
\begin{aligned}
& \ell(t)=t \quad \forall t \in[0, T / 4], \ell(t)=T-t \quad \forall t \in[3 T / 4, T], \\
& \ell(t) \leq \ell(T / 2), \forall t \in[0, T] .
\end{aligned}
$$

Then, for all $\lambda \geq 1$ and $m \geq 10$ we consider the following weight functions:

$$
\begin{aligned}
& \alpha(x, t)=\frac{\mathrm{e}^{2 \lambda\|\eta\|_{\infty}}-\mathrm{e}^{\lambda \eta(x)}}{\ell(t)^{m}}, \xi(x, t)=\frac{\mathrm{e}^{\lambda \eta(x)}}{\ell(t)^{m}}, \\
& \alpha^{*}(t)=\max _{x \in \bar{\Omega}} \alpha(x, t), \xi^{*}(t)=\min _{x \in \bar{\Omega}} \xi(x, t), \\
& \widehat{\alpha}(t)=\min _{x \in \bar{\Omega}} \alpha(x, t), \widehat{\xi}(t)=\max _{x \in \bar{\Omega}} \xi(x, t) .
\end{aligned}
$$


Notice that from (2.1), we obtain the following property:

$$
\left|\partial_{t}^{n} \partial_{x}^{l} \alpha\right|+\left|\partial_{t}^{n} \partial_{x}^{l} \xi\right| \leq C \xi^{(n+|l|+n / m)}
$$

where $n$ is any nonnegative integer, $l$ is a $N$-multi-index and $C>0$ is a constant only depending on $\Omega, \lambda, \eta$ and $\ell$. This property is also valid for the pairs $\left(\alpha^{*}, \xi^{*}\right)$ and $(\widehat{\alpha}, \widehat{\xi})$.

The first result is a Carleman inequality for the Stokes system with right-hand side in $L^{2}(Q)^{N}$ proved in [4], Proposition 2.1. This estimate has the interesting property that the local term does not contain one of the components of the solution.

Lemma 2.1. There exists a constant $\widehat{\lambda}_{0}>0$ such that for any $\lambda \geq \widehat{\lambda}_{0}$ there exists $C>0$ depending only on $\lambda$, $\Omega, \omega, \eta$ and $\ell$ such that for any $i \in\{1, \ldots, N\}$, any $g \in L^{2}(Q)^{N}$ and any $u^{0} \in H$, the solution of

$$
\begin{cases}u_{t}-\Delta u+\nabla p=g, \nabla \cdot u=0 & \text { in } Q, \\ u=0 & \text { on } \Sigma, \\ u(0)=u^{0} & \text { in } \Omega,\end{cases}
$$

satisfies

$$
\begin{aligned}
I_{1}(u):= & s^{2} \iint_{Q} \mathrm{e}^{-11 s \alpha^{*}}\left(\xi^{*}\right)^{2-2 / m}\left|u_{t}\right|^{2} \mathrm{~d} x \mathrm{~d} t+s^{3} \sum_{\substack{j=1 \\
j \neq i}}^{N} \iint_{Q} \mathrm{e}^{-2 s \alpha-9 s \alpha^{*}} \xi^{3}\left|\Delta u_{j}\right|^{2} \mathrm{~d} x \mathrm{~d} t \\
& +s^{4} \sum_{j=1, j \neq i}^{N} \iint_{Q} \mathrm{e}^{-2 s \alpha-9 s \alpha^{*}} \xi^{4}\left|\nabla u_{j}\right|^{2} \mathrm{~d} x \mathrm{~d} t+s^{4} \iint_{Q} \mathrm{e}^{-11 s \alpha^{*}}\left(\xi^{*}\right)^{4}|u|^{2} \mathrm{~d} x \mathrm{~d} t \\
\leq & C\left(\iint_{Q} \mathrm{e}^{-9 s \alpha^{*}}|g|^{2} \mathrm{~d} x \mathrm{~d} t+s^{7} \sum_{\substack{j=1 \\
j \neq i}}^{N} \iint_{\omega_{0} \times(0, T)} \mathrm{e}^{-2 s \widehat{\alpha}-9 s \alpha^{*}} \widehat{\xi}^{7}\left|u_{j}\right|^{2} \mathrm{~d} x \mathrm{~d} t\right)
\end{aligned}
$$

for every $s \geq C$.

Remark 2.2. In [4], the weight functions $\alpha$ and $\xi$ are given for $m=8$, but the proof also holds for any $m \geq 8$. Additionally, the terms involving derivatives of $u$ in the left-hand side of (2.4) do not appear explicitly in Proposition 2.1 of [4]. However, it is easily seen from its proof that these terms can be added. Finally, one should replace $\rho=\mathrm{e}^{-3 / 2 s \alpha *}$ (defined in Sect. 2.1 right after (2.13) in reference [4]) by $\rho=\mathrm{e}^{-9 / 2 s \alpha *}$.

Now, we present an estimate proved in Proposition 3.2 of [5] (with $f=0$ in that reference) similar to (2.4) with local terms of the Laplacian.

Lemma 2.3. There exists a constant $\widehat{\lambda}_{1}$, such that for any $\lambda \geq \widehat{\lambda}_{1}$ there exists a constant $C(\lambda)>0$ such that for any $i \in\{1, \ldots, N\}$, any $u^{0} \in H$ and any

$$
g \in L^{2}\left(0, T ; H^{3}(\Omega)^{N}\right) \cap H^{2}\left(0, T ; H^{-1}(\Omega)^{N}\right),
$$


the solution of (2.3) satisfies

$$
\begin{aligned}
I_{2}(u):= & \sum_{\substack{j=1 \\
j \neq i}}^{N}\left[\iint_{Q} \mathrm{e}^{-9 s \alpha}\left(s^{5} \xi^{5}\left|\Delta u_{j}\right|^{2}+s^{3} \xi^{3}\left|\nabla \Delta u_{j}\right|^{2}+s \xi\left|\nabla \nabla \Delta u_{j}\right|^{2}+s^{-1} \xi^{-1}\left|\nabla \nabla \nabla \Delta u_{j}\right|^{2}\right) \mathrm{d} x \mathrm{~d} t\right] \\
& +s^{5} \iint_{Q} \mathrm{e}^{-9 s \alpha^{*}}\left(\xi^{*}\right)^{5}|u|^{2} \mathrm{~d} x \mathrm{~d} t \leq C \int s^{5 / 2} \iint_{Q} \mathrm{e}^{-9 s \alpha^{*}}\left(\xi^{*}\right)^{3-2 / m}|g|^{2} \mathrm{~d} x \mathrm{~d} t \\
& +s^{3 / 2} \int_{0}^{T}\left\|\left(\mathrm{e}^{-9 / 2 s \alpha^{*}}\left(\xi^{*}\right)^{1-3 /(2 m)} g\right)_{t}\right\|_{H^{-1}(\Omega)^{N}}^{2} \mathrm{~d} t+s^{-1 / 2} \int_{0}^{T} \mathrm{e}^{-9 s \alpha^{*}}\left(\xi^{*}\right)^{-5 / m}\|g\|_{H^{3}(\Omega)^{N}}^{2} \mathrm{~d} t \\
& +s^{-1 / 2} \int_{0}^{T}\left\|\left(\mathrm{e}^{-9 / 2 s \alpha^{*}}\left(\xi^{*}\right)^{-5 /(2 m)} g\right)_{t}\right\|_{H^{1}(\Omega)^{N}}^{2} \mathrm{~d} t+s^{-1 / 2} \int_{0}^{T}\left\|\left(\mathrm{e}^{-9 / 2 s \alpha^{*}}\left(\xi^{*}\right)^{-5 /(2 m)} g\right)_{t t}\right\|_{H^{-1}(\Omega)^{N}}^{2} \mathrm{~d} t \\
& \left.+\sum_{j=1, j \neq i}^{N} \iint_{Q} \mathrm{e}^{-9 s \alpha}\left|\nabla \Delta g_{j}\right|^{2} \mathrm{~d} x \mathrm{~d} t+\iint_{Q} \mathrm{e}^{-9 s \alpha}|\nabla \nabla(\nabla \cdot g)|^{2} \mathrm{~d} x \mathrm{~d} t+s^{5} \sum_{\substack{j=1 \\
j \neq i}}^{N} \iint_{0} \mathrm{e}^{-9 s \alpha} \xi^{5}\left|\Delta u_{j}\right|^{2} \mathrm{~d} x \mathrm{~d} t\right)
\end{aligned}
$$

for every $s \geq C$.

The third Carleman inequality we present applies to parabolic equations with non-homogeneous boundary conditions. It was proved in ([14], Thm. 2.1):

Lemma 2.4. Let $f_{0}, f_{1}, \ldots, f_{N} \in L^{2}(Q)$. There exists a constant $\widehat{\lambda}_{2}>0$ such that for any $\lambda \geq \widehat{\lambda}_{2}$ there exists $C>0$ depending only on $\lambda, \Omega, \omega_{0}, \eta$ and $\ell$ such that for every $u \in L^{2}\left(0, T ; H^{1}(\Omega)\right) \cap H^{1}\left(0, T ; H^{-1}(\Omega)\right)$ satisfying

$$
u_{t}-\Delta u=f_{0}+\sum_{j=1}^{N} \partial_{j} f_{j} \text { in } Q,
$$

we have

$$
\begin{gathered}
s^{-1} \iint_{Q} \mathrm{e}^{-8 s \alpha} \xi^{-1}|\nabla u|^{2} \mathrm{~d} x \mathrm{~d} t+s \iint_{Q} \mathrm{e}^{-8 s \alpha} \xi|u|^{2} \mathrm{~d} x \mathrm{~d} t \leq C\left(s \iint_{\omega_{0} \times(0, T)} \mathrm{e}^{-8 s \alpha} \xi|u|^{2} \mathrm{~d} x \mathrm{~d} t\right. \\
\quad+s^{-1 / 2}\left\|\mathrm{e}^{-4 s \alpha^{*}}\left(\xi^{*}\right)^{-1 / 4} u\right\|_{H^{\frac{1}{4}, \frac{1}{2}(\Sigma)}}^{2}+s^{-1 / 2}\left\|\mathrm{e}^{-4 s \alpha^{*}}\left(\xi^{*}\right)^{-1 / 4+1 / m} u\right\|_{L^{2}(\Sigma)}^{2} \\
\left.\quad+s^{-2} \iint_{Q} \mathrm{e}^{-8 s \alpha} \xi^{-2}\left|f_{0}\right|^{2} \mathrm{~d} x \mathrm{~d} t+\sum_{j=1}^{N} \iint_{Q} \mathrm{e}^{-8 s \alpha}\left|f_{j}\right|^{2} \mathrm{~d} x \mathrm{~d} t\right),
\end{gathered}
$$

for every $s \geq C$.

Here,

$$
\|u\|_{H^{\frac{1}{4}, \frac{1}{2}(\Sigma)}}=\left(\|u\|_{H^{1 / 4}\left(0, T ; L^{2}(\partial \Omega)\right)}^{2}+\|u\|_{L^{2}\left(0, T ; H^{1 / 2}(\partial \Omega)\right)}^{2}\right)^{1 / 2} .
$$

Remark 2.5. Notice that the usual notation for this space is actually $H^{\frac{1}{2}, \frac{1}{4}}(\Sigma)$ (see, for instance, [20]). However, we choose to follow the notation used in [14]. 
The last estimate that we need is a technical result that can be found, along with its proof, in ([6], Lem. 3).

Lemma 2.6. Let $k \in \mathbb{R}$. There exists $C>0$ depending only on $\Omega, \omega_{0}, \eta$ and $\ell$ such that, for every $T>0$ and every $u \in L^{2}\left(0, T ; H^{1}(\Omega)\right)$,

$$
s^{2} \iint_{Q} \mathrm{e}^{-8 s \alpha} \xi^{k+2}|u|^{2} \mathrm{~d} x \mathrm{~d} t \leq C\left(\iint_{Q} \mathrm{e}^{-8 s \alpha} \xi^{k}|\nabla u|^{2} \mathrm{~d} x \mathrm{~d} t+s^{2} \iint_{\omega_{0} \times(0, T)} \mathrm{e}^{-8 s \alpha} \xi^{k+2}|u|^{2} \mathrm{~d} x \mathrm{~d} t\right),
$$

for every $s \geq C$.

\subsection{Regularity results}

Here, we state some regularity results concerning the heat and Stokes equations, respectively. The first one is (see for instance [17], Chapt. 4):

Lemma 2.7. For every $T>0$ and every $f \in L^{2}(Q)$, there exists a unique solution

$$
u \in L^{2}\left(0, T ; H^{2}(\Omega)\right) \cap H^{1}\left(0, T ; L^{2}(\Omega)\right)
$$

to the heat equation

$$
\begin{cases}u_{t}-\Delta u=f & \text { in } Q \\ u=0 & \text { on } \Sigma, \\ u(0)=0 & \text { in } \Omega,\end{cases}
$$

and there exists a constant $C>0$ depending only on $\Omega$ such that

$$
\|u\|_{L^{2}\left(0, T ; H^{2}(\Omega)\right)}^{2}+\|u\|_{H^{1}\left(0, T ; L^{2}(\Omega)\right)}^{2} \leq C\|f\|_{L^{2}(Q)}^{2} .
$$

Furthermore, if $f \in X_{n}$ ( $n$ any nonnegative integer), the unique solution to the heat equation (2.5) satisfies $u \in X_{n+1}$ and there exists a constant $C>0$ depending only on $\Omega$ such that

$$
\|u\|_{X_{n+1}}^{2} \leq C\|f\|_{X_{n}}^{2} \text {. }
$$

The second one can be found in ([16], Thm. 6, pp. 100-101; see also [25]):

Lemma 2.8. For every $T>0$, every $u^{0} \in V$ and every $f \in L^{2}(Q)^{N}$, there exists a unique solution

$$
u \in L^{2}\left(0, T ; H^{2}(\Omega)^{N}\right) \cap H^{1}(0, T ; H) \cap L^{\infty}(0, T ; V)
$$

to the Stokes system

$$
\begin{cases}u_{t}-\Delta u+\nabla p=f & \text { in } \quad Q, \\ \nabla \cdot u=0 & \text { in } Q, \\ u=0 & \text { on } \quad \Sigma, \\ u(0)=u^{0} & \text { in } \Omega,\end{cases}
$$

for some $p \in L^{2}\left(0, T ; H^{1}(\Omega)\right)$, and there exists a constant $C>0$ depending only on $\Omega$ such that

$$
\|u\|_{L^{2}\left(0, T ; H^{2}(\Omega)^{N}\right)}^{2}+\|u\|_{H^{1}\left(0, T ; L^{2}(\Omega)^{N}\right)}^{2}+\|u\|_{L^{\infty}(0, T ; V)}^{2} \leq C\left(\|f\|_{L^{2}(Q)^{N}}^{2}+\left\|u^{0}\right\|_{V}^{2}\right) .
$$

Moreover, if $f \in L^{2}\left(0, T ; H^{2}(\Omega)^{N}\right) \cap H^{1}\left(0, T ; L^{2}(\Omega)^{N}\right)$ and $u^{0} \in H^{3}(\Omega)^{N} \cap V$ satisfy the compatibility condition:

$$
\nabla \bar{p}=\Delta u^{0}+f(0) \quad \text { on } \quad \partial \Omega
$$

where $\bar{p}$ is any solution of the Neumann boundary-value problem

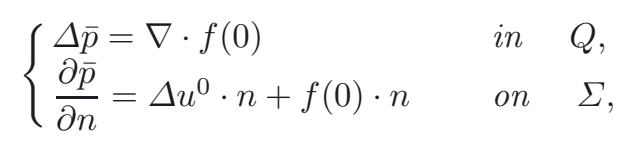


then $u \in Y_{2}$ and there exists a constant $C>0$ depending only on $\Omega$ such that

$$
\|u\|_{Y_{2}}^{2} \leq C\left(\|f\|_{Y_{1}}^{2}+\left\|u^{0}\right\|_{H^{3}(\Omega)}^{2}\right) .
$$

\section{Carleman estimate for the adjoint system}

In this section we prove a new Carleman estimate for the following coupled system:

$$
\begin{cases}-\varphi_{t}-\Delta \varphi+\nabla \pi_{\varphi}=g^{\varphi}+\psi \mathbb{1}_{\mathcal{O}}, \nabla \cdot \varphi=0 & \text { in } \quad Q, \\ \psi_{t}-\Delta \psi+\nabla \pi_{\psi}=g^{\psi}+\sigma e_{N}, \nabla \cdot \psi=0 & \text { in } \quad Q, \\ -\phi_{t}-\Delta \phi=g^{\phi}+\varphi_{N}+\sigma \mathbb{1}_{\mathcal{O}} & \text { in } \quad Q, \\ \sigma_{t}-\Delta \sigma=g^{\sigma} & \text { in } Q, \\ \varphi=\psi=0, \phi=\sigma=0 & \text { on } \Sigma, \\ \varphi(T)=0, \psi(0)=\psi^{0}, \phi(T)=0, \sigma(0)=\sigma^{0} & \text { in } \quad \Omega,\end{cases}
$$

where $g^{\varphi} \in Y_{0}, g^{\psi} \in Y_{2}, g^{\phi} \in X_{0}, g^{\sigma} \in X_{4}, \psi^{0} \in H$ and $\sigma^{0} \in L^{2}(\Omega)$. It is given by the following proposition:

Proposition 3.1. Assume that $\omega \cap \mathcal{O} \neq \emptyset$. Then, there exists a constant $\lambda_{0}$, such that for any $\lambda \geq \lambda_{0}$ there exists a constant $C>0$ depending only on $\lambda, \Omega, \omega$ and $\ell$ such that for any $j_{0} \in\{1, \ldots, N-1\}$, any $g^{\varphi} \in L^{2}(Q)^{N}$, any $g^{\psi} \in Y_{2}$, any $g^{\phi} \in L^{2}(\Omega)$, any $g^{\sigma} \in X_{4}$, any $\psi^{0} \in H$ and any $\sigma_{0} \in L^{2}(\Omega)$, the solution $(\varphi, \psi, \phi, \sigma)$ of (3.1) satisfies

$$
\begin{aligned}
& s^{4} \iint_{Q} \mathrm{e}^{-11 s \alpha^{*}}\left(\xi^{*}\right)^{4}|\varphi|^{2} \mathrm{~d} x \mathrm{~d} t+s^{5} \iint_{Q} \mathrm{e}^{-9 s \alpha^{*}}\left(\xi^{*}\right)^{5}|\psi|^{2} \mathrm{~d} x \mathrm{~d} t \\
& +s^{3} \iint_{Q} \mathrm{e}^{-12 s \alpha^{*}}\left(\xi^{*}\right)^{3}|\phi|^{2} \mathrm{~d} x \mathrm{~d} t+s^{5} \iint_{Q} \mathrm{e}^{-8 s \alpha^{*}}\left(\xi^{*}\right)^{5}|\sigma|^{2} \mathrm{~d} x \mathrm{~d} t \\
& \leq C\left(s^{15}\left\|\mathrm{e}^{-8 s \alpha+4 s \alpha^{*}} \xi^{9} g^{\varphi}\right\|_{Y_{0}}^{2}+\left\|\mathrm{e}^{-7 / 2 s \alpha^{*}} g^{\psi}\right\|_{Y_{2}}^{2}\right. \\
& +s^{19}\left\|\mathrm{e}^{-8 s \alpha+4 s \alpha^{*}} \xi^{11} g^{\phi}\right\|_{X_{0}}^{2}+\left\|\mathrm{e}^{-7 / 2 s \alpha^{*}} g^{\sigma}\right\|_{X_{4}}^{2} \\
& \left.+(N-2) s^{13} \iint_{\omega \times(0, T)} \mathrm{e}^{-9 s \alpha} \xi^{13}\left|\varphi_{j_{0}}\right|^{2} \mathrm{~d} x \mathrm{~d} t+s^{26} \iint_{\omega \times(0, T)} \mathrm{e}^{-18 s \alpha+11 s \alpha^{*}} \xi^{30}|\phi|^{2} \mathrm{~d} x \mathrm{~d} t\right),
\end{aligned}
$$

for every $s \geq C$.

For the sake of completeness, we treat the more general case of $N=3$ (with $j_{0}=1$, for instance). The general idea is to combine suitable Carleman estimates for the heat and Stokes equations in (3.1). The proof is then divided in several parts:

- First, we deduce from Lemma 2.3 a Carleman estimate for $\psi$ with local terms of $\Delta \psi_{1}$ and $\Delta \psi_{3}$. Using the coupling with the equation of $\varphi$, we estimate these terms by local terms of $\varphi_{1}$ and $\varphi_{3}$.

- Using the equation of $\phi$, we estimate the local term of $\varphi_{3}$ by a local term of $\phi$.

- Finally, to add $\sigma$ to the left hand side of (3.2) and absorb all its global terms on the right- hand side, we prove a Carleman estimate for a certain operator of $\sigma$ such that its global terms can be estimated by terms of the right-hand side of (3.2).

The details of these steps are the target of the following subsections. 


\subsection{Carleman estimate for $(\varphi, \psi)$}

The first step is to apply Lemma 2.3 to the equation satisfied by $\psi$. However, before doing that, let us remark some simple (but useful) properties of the weight functions. Note that for every $a>0$, and every $b, c \in \mathbb{R}$, the function $s^{b} \mathrm{e}^{-a s \alpha} \xi^{c}$ is bounded in $Q$. Furthermore, for any given $\varepsilon>0$, we have

$$
s^{b} \mathrm{e}^{-a s \alpha} \xi^{c}<\varepsilon,
$$

for $s \geq C, C$ a positive constant. Taking this and (2.2) into account, we have, for example,

$$
\begin{aligned}
\left|\left(s^{3 / 4} \mathrm{e}^{-9 / 2 s \alpha^{*}}\left(\xi^{*}\right)^{1-3 /(2 m)} \sigma\right)_{t}\right| & =\left|\left(s^{1 / 2} \mathrm{e}^{-4 s \alpha^{*}}\left(\xi^{*}\right)^{1 / 2-2 / m} \sigma\left(s^{1 / 4} \mathrm{e}^{-1 / 2 s \alpha^{*}}\left(\xi^{*}\right)^{1 / 2+1 /(2 m)}\right)\right)_{t}\right| \\
& \leq \varepsilon\left(\left|s^{1 / 2} \mathrm{e}^{-4 s \alpha^{*}}\left(\xi^{*}\right)^{1 / 2-2 / m} \sigma\right|+\left|\left(s^{1 / 2} \mathrm{e}^{-4 s \alpha^{*}}\left(\xi^{*}\right)^{1 / 2-2 / m} \sigma\right)_{t}\right|\right),
\end{aligned}
$$

for every $s \geq C$, that is,

$$
\left\|s^{3 / 4} \mathrm{e}^{-9 / 2 s \alpha^{*}}\left(\xi^{*}\right)^{1-3 /(2 m)} \sigma\right\|_{H^{1}\left(L^{2}\right)} \leq \varepsilon\left\|s^{1 / 2} \mathrm{e}^{-4 s \alpha^{*}}\left(\xi^{*}\right)^{1 / 2-2 / m} \sigma\right\|_{H^{1}\left(L^{2}\right)} .
$$

\subsubsection{Carleman estimate for $\psi$}

Now, we apply Lemma 2.3 with $i=2, u=\psi$, and $g=g^{\psi}+\sigma e_{3}$. Using (3.3) and the idea developed in (3.4) we obtain

$$
\begin{aligned}
I_{2}(\psi) & \leq C\left\|\mathrm{e}^{-7 / 2 s \alpha^{*}} g^{\psi}\right\|_{Y_{2}}^{2}+\varepsilon\left\|s^{1 / 2} \mathrm{e}^{-4 s \alpha^{*}}\left(\xi^{*}\right)^{1 / 2-2 / m} \sigma\right\|_{X_{2}}^{2} \\
& +C s^{5} \iint_{\omega_{0} \times(0, T)} \mathrm{e}^{-9 s \alpha} \xi^{5}\left(\left|\Delta \psi_{1}\right|^{2}+\left|\Delta \psi_{3}\right|^{2}\right) \mathrm{d} x \mathrm{~d} t,
\end{aligned}
$$

for every $s \geq C, \varepsilon>0$ to be chosen small enough.

\subsubsection{Carleman estimate for $\varphi$}

Next, we apply Lemma 2.1 to the equation satisfied by $\varphi$, with $i=2, u=\varphi$ and $g=g^{\varphi}+\psi \mathbb{1}_{\mathcal{O}}$. We obtain

$$
\begin{aligned}
I_{1}(\varphi) \leq & C \iint_{Q} \mathrm{e}^{-9 s \alpha^{*}}\left|g^{\varphi}\right|^{2} \mathrm{~d} x \mathrm{~d} t+C \iint_{\mathcal{O} \times(0, T)} \mathrm{e}^{-9 s \alpha^{*}}|\psi|^{2} \mathrm{~d} x \mathrm{~d} t \\
& +C s^{7} \iint_{\omega_{0} \times(0, T)} \mathrm{e}^{-2 s \alpha-9 s \alpha^{*}} \xi^{7}\left(\left|\varphi_{1}\right|^{2}+\left|\varphi_{3}\right|^{2}\right) \mathrm{d} x \mathrm{~d} t,
\end{aligned}
$$

for every $s \geq C$. Noticing that the second integral in the right of this inequality is bounded by $C s^{-5} I_{2}(\psi)$, for some $C>0$, we can combine inequalities (3.5) and (3.6) to get

$$
\begin{aligned}
I_{1}(\varphi)+I_{2}(\psi) \leq & C\left\|\mathrm{e}^{-9 / 2 s \alpha^{*}} g^{\varphi}\right\|_{Y_{0}}^{2}+C\left\|\mathrm{e}^{-7 / 2 s \alpha^{*}} g^{\psi}\right\|_{Y_{2}}^{2}+\varepsilon\left\|s^{1 / 2} \mathrm{e}^{-4 s \alpha^{*}}\left(\xi^{*}\right)^{1 / 2-2 / m} \sigma\right\|_{X_{2}}^{2} \\
& +C s^{7} \iint_{\omega_{0} \times(0, T)} \mathrm{e}^{-2 s \alpha-9 s \alpha^{*}} \xi^{7}\left(\left|\varphi_{1}\right|^{2}+\left|\varphi_{3}\right|^{2}\right) \mathrm{d} x \mathrm{~d} t \\
& +C s^{5} \iint_{\omega_{0} \times(0, T)} \mathrm{e}^{-9 s \alpha} \xi^{5}\left(\left|\Delta \psi_{1}\right|^{2}+\left|\Delta \psi_{3}\right|^{2}\right) \mathrm{d} x \mathrm{~d} t,
\end{aligned}
$$

for every $s \geq C$. Now, using the coupling between $\varphi$ and $\psi$ we will estimate the last two local terms. This is the objective of the next subsection. 


\subsubsection{Estimation of $\Delta \psi_{1}$ and $\Delta \psi_{3}$}

We look at the equations satisfied by $\varphi_{1}$ and $\varphi_{3}$ in the set $\mathcal{O} \times(0, T)$ to find

$$
\begin{aligned}
& \Delta \psi_{1}=-\Delta \varphi_{1, t}-\Delta^{2} \varphi_{1}+\partial_{1} \nabla \cdot g^{\varphi}-\Delta g_{1}^{\varphi}, \\
& \Delta \psi_{3}=-\Delta \varphi_{3, t}-\Delta^{2} \varphi_{3}+\partial_{3} \nabla \cdot g^{\varphi}-\Delta g_{3}^{\varphi} .
\end{aligned}
$$

Here, we have used that $\Delta \pi_{\varphi}=\nabla \cdot g^{\varphi}$ in $\mathcal{O} \times(0, T)$ which follows directly from the free-divergence conditions for $\varphi$ and $\psi$. Let now $\zeta \in \mathcal{C}_{0}^{4}(\widetilde{\omega})$ be a nonnegative function with $\zeta \equiv 1$ in $\omega_{0}$ and $\omega_{0} \Subset \widetilde{\omega} \Subset \omega \cap \mathcal{O}$.

For simplicity, we only treat the term concerning $\varphi_{3}$ in the last integral in (3.7), since the other term is quite similar. Using this last equality, we have

$$
\begin{aligned}
& s^{5} \iint_{\omega_{0} \times(0, T)} \mathrm{e}^{-9 s \alpha} \xi^{5}\left|\Delta \psi_{3}\right|^{2} \mathrm{~d} x \mathrm{~d} t \leq s^{5} \iint_{\widetilde{\omega} \times(0, T)} \zeta(x) \mathrm{e}^{-9 s \alpha} \xi^{5}\left|\Delta \psi_{3}\right|^{2} \mathrm{~d} x \mathrm{~d} t \\
& =s^{5} \iint_{\widetilde{\omega} \times(0, T)} \zeta(x) \mathrm{e}^{-9 s \alpha} \xi^{5} \Delta \psi_{3}\left(-\Delta \varphi_{3, t}-\Delta^{2} \varphi_{3}+\partial_{3} \nabla \cdot g^{\varphi}-\Delta g_{3}^{\varphi}\right) \mathrm{d} x \mathrm{~d} t .
\end{aligned}
$$

An integration by parts, in time and space, gives the following inequality:

$$
\begin{aligned}
& \quad s^{5} \iint_{\omega_{0} \times(0, T)} \mathrm{e}^{-9 s \alpha} \xi^{5}\left|\Delta \psi_{3}\right|^{2} \mathrm{~d} x \mathrm{~d} t \\
& \leq s^{5} \iint_{\widetilde{\omega} \times(0, T)} \zeta(x) \mathrm{e}^{-9 s \alpha} \xi^{5}\left(\Delta \psi_{3, t}-\Delta^{2} \psi_{3}\right) \Delta \varphi_{3} \mathrm{~d} x \mathrm{~d} t \\
& \quad+s^{5} \iint_{\widetilde{\omega} \times(0, T)} \Delta\left(\left(\zeta(x) \mathrm{e}^{-9 s \alpha} \xi^{5}\right)_{t} \Delta \psi_{3}-\Delta\left(\zeta(x) \mathrm{e}^{-9 s \alpha} \xi^{5}\right) \Delta \psi_{3}-2 \nabla\left(\zeta(x) \mathrm{e}^{-9 s \alpha} \xi^{5}\right) \cdot \nabla \Delta \psi_{3}\right) \varphi_{3} \mathrm{~d} x \mathrm{~d} t \\
& +s^{5} \iint_{\widetilde{\omega} \times(0, T)} \nabla \partial_{3}\left(\zeta(x) \mathrm{e}^{-9 s \alpha} \xi^{5} \Delta \psi_{3}\right) \cdot g^{\varphi} \mathrm{d} x \mathrm{~d} t-s^{5} \iint_{\widetilde{\omega} \times(0, T)} \Delta\left(\zeta(x) \mathrm{e}^{-9 s \alpha} \xi^{5} \Delta \psi_{3}\right) g_{3}^{\varphi} \mathrm{d} x \mathrm{~d} t .
\end{aligned}
$$

Now we estimate the three lines of terms in the right-hand side of (3.8), which we call respectively $L_{1}, L_{2}$ and $L_{3}$. For the first one, we use the equation satisfied by $\Delta \psi_{3}$, namely

$$
\Delta \psi_{3, t}-\Delta^{2} \psi_{3}=\Delta g_{3}^{\psi}-\partial_{3} \nabla \cdot g^{\psi}+\left(\partial_{1}^{2}+\partial_{2}^{2}\right) \sigma .
$$

This equation, together with some integration by parts, yields

$$
L_{1}=s^{5} \iint_{\widetilde{\omega} \times(0, T)} \Delta\left(\zeta(x) \mathrm{e}^{-9 s \alpha} \xi^{5}\left(\Delta g_{3}^{\psi}-\partial_{3} \nabla \cdot g^{\psi}+\left(\partial_{1}^{2}+\partial_{2}^{2}\right) \sigma\right)\right) \varphi_{3} \mathrm{~d} x \mathrm{~d} t .
$$

A careful analysis of these terms, taking into account (2.2), (3.3) and using Young's inequality, gives

$$
\left|L_{1}\right| \leq C s^{13} \iint_{\widetilde{\omega} \times(0, T)} \mathrm{e}^{-9 s \alpha} \xi^{13}\left|\varphi_{3}\right|^{2} \mathrm{~d} x \mathrm{~d} t+C\left\|\mathrm{e}^{-7 / 2 s \alpha^{*}} g^{\psi}\right\|_{Y_{2}}^{2}+\varepsilon\left\|s^{1 / 2} \mathrm{e}^{-4 s \alpha^{*}}\left(\xi^{*}\right)^{1 / 2-2 / m} \sigma\right\|_{X_{2}}^{2} .
$$


A similar argument for $L_{2}$ and $L_{3}$ gives the following estimates

$$
\begin{aligned}
& \left|L_{2}\right| \leq \frac{1}{4 C} I_{2}(\psi)+C s^{13} \iint_{\widetilde{\omega} \times(0, T)} \mathrm{e}^{-9 s \alpha} \xi^{13}\left|\varphi_{3}\right|^{2} \mathrm{~d} x \mathrm{~d} t, \\
& \left|L_{3}\right| \leq \frac{1}{4 C} I_{2}(\psi)+C s^{9} \iint_{\widetilde{\omega} \times(0, T)} \mathrm{e}^{-9 s \alpha} \xi^{9}\left|g^{\varphi}\right|^{2} \mathrm{~d} x \mathrm{~d} t .
\end{aligned}
$$

Combining these estimates for $L_{1}, L_{2}$ and $L_{3}$ with (3.8), together with the same computations for $\psi_{1}$, provide

$$
\begin{aligned}
I_{1}(\varphi)+I_{2}(\psi) & \leq C\left(\left\|s^{9 / 2} \mathrm{e}^{-9 / 2 s \alpha} \xi^{9 / 2} g^{\varphi}\right\|_{Y_{0}}^{2}+\left\|\mathrm{e}^{-7 / 2 s \alpha^{*}} g^{\psi}\right\|_{Y_{2}}^{2}\right) \\
& +\varepsilon\left\|s^{1 / 2} \mathrm{e}^{-4 s \alpha^{*}}\left(\xi^{*}\right)^{1 / 2-2 / m} \sigma\right\|_{X_{2}}^{2} \\
& +C s^{13} \iint_{\widetilde{\omega} \times(0, T)} \mathrm{e}^{-9 s \alpha} \xi^{13}\left(\left|\varphi_{1}\right|^{2}+\left|\varphi_{3}\right|^{2}\right) \mathrm{d} x \mathrm{~d} t
\end{aligned}
$$

for every $s \geq C$.

Here, we have also used the properties of the weight functions to find a more compact expression. This ends this part of the proof of Proposition 3.1.

The next step is to eliminate the local term of $\varphi_{3}$. This is done in the next subsection.

\subsection{Estimation of $\varphi_{3}$ and Carleman estimate for $\phi$}

As in the previous section, we look at the equation satisfied by $\phi$ in the set $\mathcal{O} \times(0, T)$ :

$$
\varphi_{3}=-\phi_{t}-\Delta \phi-g^{\phi}-\sigma .
$$

We consider again a nonnegative function $\zeta \in \mathcal{C}_{0}^{2}\left(\omega^{\prime}\right)$ such that $\zeta \equiv 1$ in $\widetilde{\omega}$ and $\widetilde{\omega} \Subset \omega^{\prime} \Subset \omega \cap \mathcal{O}$, and perform integration by parts to obtain

$$
\begin{aligned}
s^{13} \iint_{\omega^{\prime} \times(0, T)} \zeta(x) \mathrm{e}^{-9 s \alpha} \xi^{13}\left|\varphi_{3}\right|^{2} \mathrm{~d} x \mathrm{~d} t= & s^{13} \iint_{\omega^{\prime} \times(0, T)} \zeta(x) \mathrm{e}^{-9 s \alpha} \xi^{13} \varphi_{3}\left(-\phi_{t}-\Delta \phi-g^{\phi}-\sigma\right) \mathrm{d} x \mathrm{~d} t \\
= & s^{13} \iint_{\omega^{\prime} \times(0, T)}\left[\partial_{t}-\Delta\right]\left(\zeta(x) \mathrm{e}^{-9 s \alpha} \xi^{13} \varphi_{3}\right) \phi \mathrm{d} x \mathrm{~d} t \\
& -s^{13} \iint_{\omega^{\prime} \times(0, T)} \zeta(x) \mathrm{e}^{-9 s \alpha} \xi^{13} \varphi_{3}\left(g^{\phi}+\sigma\right) \mathrm{d} x \mathrm{~d} t .
\end{aligned}
$$

Let us call $L_{4}$ and $L_{5}$ the two integrals at the end of the last expression. A careful analysis of $L_{4}$, together with property (2.2) and Young's inequality, yield

$$
\left|L_{4}\right| \leq \frac{1}{2 C} I_{1}(\varphi)+C s^{26} \iint_{\omega^{\prime} \times(0, T)} \mathrm{e}^{-18 s \alpha+11 s \alpha^{*}} \xi^{30}|\phi|^{2} \mathrm{~d} x \mathrm{~d} t+C s^{24} \iint_{\omega^{\prime} \times(0, T)} \mathrm{e}^{-16 s \alpha+9 s \alpha^{*}} \xi^{24}|\phi|^{2} \mathrm{~d} x \mathrm{~d} t,
$$

for every $s \geq C$. 
For the term concerning $L_{5}$, we apply again Young's inequality and property (3.3):

$$
\begin{aligned}
\left|L_{5}\right| \leq & \frac{1}{2 C} s^{13} \iint_{\omega^{\prime} \times(0, T)} \zeta(x) \mathrm{e}^{-9 s \alpha} \xi^{13}\left|\varphi_{3}\right|^{2} \mathrm{~d} x \mathrm{~d} t+C\left\|s^{13 / 2} \mathrm{e}^{-9 / 2 s \alpha} \xi^{13 / 2} g^{\phi}\right\|_{X_{0}}^{2} \\
& +\varepsilon\left\|s^{1 / 2} \mathrm{e}^{-4 s \alpha^{*}}\left(\xi^{*}\right)^{1 / 2-2 / m} \sigma\right\|_{X_{0}}^{2} .
\end{aligned}
$$

From (2.1), we have $\mathrm{e}^{-16 s \alpha+9 s \alpha^{*}}<\mathrm{e}^{-18 s \alpha+11 s \alpha^{*}}$ in $Q$, so we obtain

$$
\begin{aligned}
s^{13} \iint_{\omega^{\prime} \times(0, T)} \zeta(x) \mathrm{e}^{-9 s \alpha} \xi^{13}\left|\varphi_{3}\right|^{2} \mathrm{~d} x \mathrm{~d} t \leq & \frac{1}{2 C} I_{1}(\varphi)+C s^{26} \iint_{\omega^{\prime} \times(0, T)} \mathrm{e}^{-18 s \alpha+11 s \alpha^{*}} \xi^{30}|\phi|^{2} \mathrm{~d} x \mathrm{~d} t \\
& +C\left\|s^{13 / 2} \mathrm{e}^{-9 / 2 s \alpha} \xi^{13 / 2} g^{\phi}\right\|_{X_{0}}^{2} \\
& +\varepsilon\left\|s^{1 / 2} \mathrm{e}^{-4 s \alpha^{*}}\left(\xi^{*}\right)^{1 / 2-2 / m} \sigma\right\|_{X_{0}}^{2},
\end{aligned}
$$

for every $s \geq C$, which plugged in (3.10) together with the fact that

$$
s^{13} \iint_{\widetilde{\omega} \times(0, T)} \mathrm{e}^{-9 s \alpha} \xi^{13}\left|\varphi_{3}\right|^{2} \mathrm{~d} x \mathrm{~d} t \leq s^{13} \iint_{\omega^{\prime} \times(0, T)} \zeta(x) \mathrm{e}^{-9 s \alpha} \xi^{13}\left|\varphi_{3}\right|^{2} \mathrm{~d} x \mathrm{~d} t,
$$

leads to

$$
\begin{aligned}
I_{1}(\varphi)+I_{2}(\psi) \leq & C\left\|s^{9 / 2} \mathrm{e}^{-9 / 2 s \alpha} \xi^{9 / 2} g^{\varphi}\right\|_{Y_{0}}^{2}+C\left\|\mathrm{e}^{-7 / 2 s \alpha^{*}} g^{\psi}\right\|_{Y_{2}}^{2}+C\left\|s^{13 / 2} \mathrm{e}^{-9 / 2 s \alpha} \xi^{13 / 2} g^{\phi}\right\|_{X_{0}}^{2} \\
& +\varepsilon\left\|s^{1 / 2} \mathrm{e}^{-4 s \alpha^{*}}\left(\xi^{*}\right)^{1 / 2-2 / m} \sigma\right\|_{X_{2}}^{2}+C s^{13} \iint_{\widetilde{\omega} \times(0, T)} \mathrm{e}^{-9 s \alpha} \xi^{13}\left|\varphi_{1}\right|^{2} \mathrm{~d} x \mathrm{~d} t \\
& +C s^{26} \iint_{\omega^{\prime} \times(0, T)} \mathrm{e}^{-18 s \alpha+11 s \alpha^{*}} \xi^{30}|\phi|^{2} \mathrm{~d} x \mathrm{~d} t,
\end{aligned}
$$

for every $s \geq C$.

To end this section of the proof, we will combine (3.11) with a Carleman inequality for $\phi$. This will allow us to add the term with $\phi$ in the left hand side of (3.2). Namely, $\phi$ satisfies

$$
\begin{aligned}
I_{3}(\phi):= & \iint_{Q} \mathrm{e}^{-12 s \alpha}\left(s^{3} \xi^{3}|\phi|^{2}+s \xi|\nabla \phi|^{2}+s^{-1} \xi^{-1}\left(\left|\phi_{t}\right|^{2}+|\Delta \phi|^{2}\right)\right) \mathrm{d} x \mathrm{~d} t \\
& \leq C \iint_{Q} \mathrm{e}^{-12 s \alpha}\left(\left|g^{\phi}\right|^{2}+\left|\varphi_{3}\right|^{2}+|\sigma|^{2} \mathbb{1}_{\mathcal{O}}\right) \mathrm{d} x \mathrm{~d} t+C s^{3} \iint_{\omega \times(0, T)} \mathrm{e}^{-12 s \alpha} \xi^{3}|\phi|^{2} \mathrm{~d} x \mathrm{~d} t
\end{aligned}
$$

for every $s \geq C$. This is the classical Carleman estimate for the solutions of the heat equation with homogeneous Dirichlet boundary conditions (see, for instance, [10]). Notice that, taking (3.3) into account, the right-hand side of (3.12) is bounded by

$$
\begin{aligned}
C\left\|s^{13 / 2} \mathrm{e}^{-9 / 2 s \alpha} \xi^{13 / 2} g^{\phi}\right\|_{X_{0}}^{2} & +\frac{1}{2} I_{1}(\varphi)+\varepsilon\left\|s^{1 / 2} \mathrm{e}^{-4 s \alpha^{*}}\left(\xi^{*}\right)^{1 / 2-2 / m} \sigma\right\|_{X_{2}}^{2} \\
& +C s^{26} \iint_{\omega \times(0, T)} \mathrm{e}^{-18 s \alpha+11 s \alpha^{*}} \xi^{30}|\phi|^{2} \mathrm{~d} x \mathrm{~d} t,
\end{aligned}
$$


for every $s \geq C$. Thus, combining this bound with (3.11) and (3.12) we obtain

$$
\begin{aligned}
I_{1}(\varphi)+I_{2}(\psi)+I_{3}(\phi) \leq & C\left(\left\|s^{9 / 2} \mathrm{e}^{-9 / 2 s \alpha} \xi^{9 / 2} g^{\varphi}\right\|_{Y_{0}}^{2}+\left\|\mathrm{e}^{-7 / 2 s \alpha^{*}} g^{\psi}\right\|_{Y_{2}}^{2}\right. \\
& \left.+\left\|s^{13 / 2} \mathrm{e}^{-9 / 2 s \alpha} \xi^{13 / 2} g^{\phi}\right\|_{X_{0}}^{2}\right)+\varepsilon\left\|s^{1 / 2} \mathrm{e}^{-4 s \alpha^{*}}\left(\xi^{*}\right)^{1 / 2-2 / m} \sigma\right\|_{X_{2}}^{2} \\
& +C s^{13} \iint_{\omega \times(0, T)} \mathrm{e}^{-9 s \alpha} \xi^{13}\left|\varphi_{1}\right|^{2} \mathrm{~d} x \mathrm{~d} t+C s^{26} \iint_{\omega \times(0, T)} \mathrm{e}^{-18 s \alpha+11 s \alpha^{*}} \xi^{30}|\phi|^{2} \mathrm{~d} x \mathrm{~d} t,
\end{aligned}
$$

for every $s \geq C$.

At this point, there are a few tasks left to do. First, we need to eliminate the global term of $\sigma$ in the right-hand side of (3.13). This will be done combining this estimate with a suitable Carleman estimate for $\sigma$, provided that we choose the weight functions conveniently. This will add $\sigma$ to the left-hand side and a local term of $\sigma$ to the right-hand side that also needs to be eliminated. Normally, we would try to use again the coupling of $\sigma$ with the other equations. However, this will make appear the pressure term of the equation of $\psi$, or again a local term of $\varphi_{3}$ in the equation of $\phi$, which we would not be able to eliminate again. Therefore, a more careful analysis needs to be done to avoid these issues. Details are given in the next subsection.

\subsection{Carleman estimate for $\sigma$ and final computations}

This final part of the proof of Proposition 3.1 is divided in three steps. In the first one, we look for an equation of $\sigma$ which does not contain neither $\pi_{\psi}$ nor $\varphi_{3}$. Then, we show a suitable Carleman inequality for $\sigma$ based on this expression. Finally, we eliminate the local terms of $\sigma$ and conclude.

\subsubsection{Operator for $\sigma$}

Let us look at system (3.1) in the set $\mathcal{O} \times(0, T)$. All the following computations will be seen in this set. Since $\Delta \pi_{\varphi}=\nabla \cdot g^{\varphi}$, we find

$$
-\left(\Delta \varphi_{3}\right)_{t}-\Delta^{2} \varphi_{3}=\Delta g_{3}^{\varphi}-\partial_{3} \nabla \cdot g^{\varphi}+\Delta \psi_{3} .
$$

In the previous section, we found the equation satisfied by $\Delta \psi_{3}$ (see (3.9))

$$
\left(\Delta \psi_{3}\right)_{t}-\Delta^{2} \psi_{3}=\Delta g_{3}^{\psi}-\partial_{3} \nabla \cdot g^{\psi}+\mathcal{D} \sigma
$$

Here, we recall the notation used in (1.9), $\mathcal{D}:=\partial_{1}^{2}+\partial_{2}^{2}$. Combining these expressions, we can easily find the following relation between $\varphi_{3}$ and $\sigma$ :

$$
\mathcal{P} \varphi_{3}=\left(\Delta g_{3}^{\varphi}\right)_{t}-\Delta^{2} g_{3}^{\varphi}-\left(\partial_{3} \nabla \cdot g^{\varphi}\right)_{t}+\Delta\left(\partial_{3} \nabla \cdot g^{\varphi}\right)+\Delta g_{3}^{\psi}-\partial_{3} \nabla \cdot g^{\psi}+\mathcal{D} \sigma
$$

where we have denoted $\mathcal{P}=-\Delta \partial_{t}^{2}+\Delta^{3}$ (recall again (1.9)).

Finally, we apply this operator to the equation satisfied by $\phi$ and, combined with the last expression, we obtain

$$
\begin{aligned}
-\left(\mathcal{P}_{t}+\Delta \mathcal{P}\right) \phi= & \left(\Delta g_{3}^{\varphi}\right)_{t}-\Delta^{2} g_{3}^{\varphi}-\partial_{3} \nabla \cdot g_{t}^{\varphi}+\Delta\left(\partial_{3} \nabla \cdot g^{\varphi}\right)+\Delta g_{3}^{\psi}-\partial_{3} \nabla \cdot g^{\psi} \\
& +\mathcal{P} g^{\phi}-\Delta g_{t}^{\sigma}-\Delta^{2} g^{\sigma}+\mathcal{D} \sigma
\end{aligned}
$$

where we have used the equation satisfied by $\sigma$ to find $\mathcal{P} \sigma=-\Delta g_{t}^{\sigma}-\Delta^{2} g^{\sigma}$.

The idea now is to prove a Carleman estimate for $\sigma$ with a local term of $\mathcal{D} \sigma$ and use (3.14) to eliminate it. 


\subsubsection{Carleman estimate for $\mathcal{D} \sigma$}

Here, we follow the ideas developed for the Stokes system in [6] (see also $[4,5]$ ), thus for simplicity we omit some detail of the computations. We start by applying $\nabla \nabla \mathcal{D}$ to the equation satisfied by $\sigma$. We have

$$
(\nabla \nabla \mathcal{D} \sigma)_{t}-\Delta(\nabla \nabla \mathcal{D} \sigma)=\nabla \nabla \mathcal{D} g^{\sigma}, \text { in } Q
$$

We apply sequentially to this equation:

- Lemma 2.4 with $u=\nabla \nabla \mathcal{D} \sigma$,

- Lemma 2.6 with $k=1$ and $u=\nabla \mathcal{D} \sigma$,

- Lemma 2.6 with $k=3$ and $u=\mathcal{D} \sigma$,

and

$$
\int_{\Omega}|\sigma|^{2} \mathrm{~d} x \leq C \int_{\Omega}|\mathcal{D} \sigma|^{2} \mathrm{~d} x,
$$

for some $C$ depending on $\Omega$ (this last inequality holds since $\Omega$ is bounded and $\sigma_{\mid \partial \Omega}=0$ ). Therefore, we obtain

$$
\begin{aligned}
\widetilde{I}_{4}(\sigma):= & \iint_{Q} \mathrm{e}^{-8 s \alpha}\left(s^{-1} \xi^{-1}\left|\nabla^{3} \mathcal{D} \sigma\right|^{2}+s \xi\left|\nabla^{2} \mathcal{D} \sigma\right|^{2}+s^{3} \xi^{3}|\nabla \mathcal{D} \sigma|^{2}+s^{5} \xi^{5}|\mathcal{D} \sigma|^{2}\right) \mathrm{d} x \mathrm{~d} t \\
& +s^{5} \iint_{Q} \mathrm{e}^{-8 s \alpha^{*}}\left(\xi^{*}\right)^{5}|\sigma|^{2} \mathrm{~d} x \mathrm{~d} t \leq C s^{-2} \iint_{Q} \mathrm{e}^{-8 s \alpha} \xi^{-2}\left|\nabla^{2} \mathcal{D} g^{\sigma}\right|^{2} \mathrm{~d} x \mathrm{~d} t \\
& +C s^{-1 / 2}\left\|\mathrm{e}^{-4 s \alpha^{*}}\left(\xi^{*}\right)^{1 / 4} \nabla^{2} \mathcal{D} \sigma\right\|_{H^{1 / 4,1 / 2}(\Sigma)}^{2}+C s^{-1 / 2}\left\|\mathrm{e}^{-4 s \alpha^{*}}\left(\xi^{*}\right)^{-1 / 4+1 / m} \nabla^{2} \mathcal{D} \sigma\right\|_{L^{2}(\Sigma)}^{2} \\
& +C \iint_{\omega_{0} \times(0, T)} \mathrm{e}^{-8 s \alpha}\left(s \xi\left|\nabla^{2} \mathcal{D} \sigma\right|^{2}+s^{3} \xi^{3}|\nabla \mathcal{D} \sigma|^{2}+s^{5} \xi^{5}|\mathcal{D} \sigma|^{2}\right) \mathrm{d} x \mathrm{~d} t .
\end{aligned}
$$

It is not hard to prove that, considering a cut-off function supported in $\omega^{\prime}$ (recall that $\omega_{0} \Subset \omega^{\prime} \Subset \omega \cap \mathcal{O}$ ); integration by parts and Young's inequality, we can estimate the local terms in the last inequality by

$$
\frac{1}{2} \widetilde{I}_{4}(\sigma)+C s^{5} \iint_{\omega^{\prime} \times(0, T)} \mathrm{e}^{-8 s \alpha} \xi^{5}|\mathcal{D} \sigma|^{2} \mathrm{~d} x \mathrm{~d} t .
$$

To estimate the boundary terms, we use regularity results for the heat equation. We start by defining

$$
\widetilde{\sigma}:=s^{3 / 2} \mathrm{e}^{-4 s \alpha^{*}}\left(\xi^{*}\right)^{3 / 2-1 / m} \sigma .
$$

It is not difficult to check that $\widetilde{\sigma}$ satisfies the heat equation

$$
\begin{cases}\widetilde{\sigma}_{t}-\Delta \widetilde{\sigma}=s^{3 / 2} \mathrm{e}^{-4 s \alpha^{*}}\left(\xi^{*}\right)^{3 / 2-1 / m} g^{\sigma}+\left(s^{3 / 2} \mathrm{e}^{-4 s \alpha^{*}}\left(\xi^{*}\right)^{3 / 2-1 / m}\right)_{t} \sigma & \text { in } Q, \\ \widetilde{\sigma}=0 & \text { on } \Sigma, \\ \widetilde{\sigma}(0)=0 & \text { on } \Omega .\end{cases}
$$

From regularity result (2.6) and (2.2), we obtain

$$
\|\widetilde{\sigma}\|_{X_{1}}^{2} \leq C\left(\left\|s^{3 / 2} \mathrm{e}^{-4 s \alpha^{*}}\left(\xi^{*}\right)^{3 / 2-1 / m} g^{\sigma}\right\|_{X_{0}}^{2}+\left\|s^{5 / 2} \mathrm{e}^{-4 s \alpha^{*}}\left(\xi^{*}\right)^{5 / 2} \sigma\right\|_{X_{0}}^{2}\right) .
$$


Applying successively regularity result (2.7), taking into account (2.2), we can prove the general formula

$$
\begin{aligned}
\left\|s^{5 / 2-n} \mathrm{e}^{-4 s \alpha^{*}}\left(\xi^{*}\right)^{5 / 2-n-n / m} \sigma\right\|_{X_{n}}^{2} \leq & C \sum_{k=0}^{n-1}\left\|s^{3 / 2-k} \mathrm{e}^{-4 s \alpha^{*}}\left(\xi^{*}\right)^{3 / 2-k-(k+1) / m} g^{\sigma}\right\|_{X_{k}}^{2} \\
& +C\left\|s^{5 / 2} \mathrm{e}^{-4 s \alpha^{*}}\left(\xi^{*}\right)^{5 / 2} \sigma\right\|_{X_{0}}^{2},
\end{aligned}
$$

for $n=1, \ldots, 5$. Let us call in what follows

$$
\mathcal{R}_{n}\left(g^{\sigma}\right):=\sum_{k=0}^{n-1}\left\|s^{3 / 2-k} \mathrm{e}^{-4 s \alpha^{*}}\left(\xi^{*}\right)^{3 / 2-k-(k+1) / m} g^{\sigma}\right\|_{X_{k}}^{2} .
$$

Using a trace inequality (see, for instance, [20]) we have

$$
\left\|\mathrm{e}^{-4 s \alpha^{*}}\left(\xi^{*}\right)^{1 / 4} \nabla^{2} \mathcal{D} \sigma\right\|_{H^{1 / 4,1 / 2}(\Sigma)}^{2} \leq C\left\|\mathrm{e}^{-4 s \alpha^{*}}\left(\xi^{*}\right)^{1 / 4} \sigma\right\|_{L^{2}\left(0, T ; H^{5}(\Omega)\right) \cap H^{1}\left(0, T ; H^{3}(\Omega)\right)}^{2} .
$$

Now, by an interpolation argument between the spaces $X_{2}$ and $X_{3}$, and since $m \geq 10$, we can combine this estimate with (3.18) and write

$$
s^{-1 / 2}\left\|\mathrm{e}^{-4 s \alpha^{*}}\left(\xi^{*}\right)^{1 / 4} \nabla^{2} \mathcal{D} \sigma\right\|_{H^{1 / 4,1 / 2}(\Sigma)}^{2} \leq C s^{-1 / 2} \mathcal{R}_{3}\left(g^{\sigma}\right)+C s^{-1 / 2}\left\|s^{5 / 2} \mathrm{e}^{-4 s \alpha^{*}}\left(\xi^{*}\right)^{5 / 2} \sigma\right\|_{X_{0}}^{2} .
$$

For the other boundary term, taking into account that $\alpha^{*}$ and $\xi^{*}$ do not depend on $x$, and $\left(\xi^{*}\right)^{-1 / 4+1 / m}$ is bounded since $m \geq 10$, we can easily obtain

$$
\begin{aligned}
& s^{-1 / 2}\left\|\mathrm{e}^{-4 s \alpha^{*}}\left(\xi^{*}\right)^{-1 / 4+1 / m} \nabla^{2} \mathcal{D} \sigma\right\|_{L^{2}(\Sigma)}^{2} \leq C s^{-1 / 2}\left\|\mathrm{e}^{-4 s \alpha^{*}} \nabla^{2} \mathcal{D} \sigma\right\|_{L^{2}(\Sigma)}^{2} \\
& \leq C s^{-1 / 2}\left(\left\|s^{1 / 2} \mathrm{e}^{-4 s \alpha^{*}}\left(\xi^{*}\right)^{1 / 2} \nabla^{2} \mathcal{D} \sigma\right\|_{L^{2}(Q)}^{2}\left\|s^{-1 / 2} \mathrm{e}^{-4 s \alpha^{*}}\left(\xi^{*}\right)^{-1 / 2} \nabla^{3} \mathcal{D} \sigma\right\|_{L^{2}(Q)}^{2}\right. \\
& \left.\quad+\left\|\mathrm{e}^{-4 s \alpha^{*}} \nabla^{2} \mathcal{D} \sigma\right\|_{L^{2}(Q)}^{2}\right) \leq C s^{-1 / 2} \widetilde{I}_{4}(\sigma)
\end{aligned}
$$

Finally, notice that from (3.18), we have

$$
J(\sigma):=\sum_{k=1}^{5}\left\|s^{5 / 2-k} \mathrm{e}^{-4 s \alpha^{*}}\left(\xi^{*}\right)^{5 / 2-k-k / m} \sigma\right\|_{X_{k}}^{2} \leq C \mathcal{R}_{5}\left(g^{\sigma}\right)+C \widetilde{I}_{4}(\sigma) .
$$

Combining estimates (3.17), (3.19)-(3.21) in (3.16), we obtain the following Carleman estimate for $\sigma$ :

$$
\begin{aligned}
I_{4}(\sigma):= & \widetilde{I}_{4}(\sigma)+J(\sigma) \leq C s^{-2} \iint_{Q} \mathrm{e}^{-8 s \alpha} \xi^{-2}\left|\nabla^{2} \mathcal{D} g^{\sigma}\right|^{2} \mathrm{~d} x \mathrm{~d} t+C \mathcal{R}_{5}\left(g^{\sigma}\right) \\
& +C s^{5} \iint_{\omega^{\prime} \times(0, T)} \mathrm{e}^{-8 s \alpha} \xi^{5}|\mathcal{D} \sigma|^{2} \mathrm{~d} x \mathrm{~d} t
\end{aligned}
$$

for every $s \geq C$. Furthermore, by (2.2), (3.3) and (3.4), we find the more compact form:

$$
I_{4}(\sigma) \leq C\left\|\mathrm{e}^{-7 / 2 s \alpha^{*}} g^{\sigma}\right\|_{X_{4}}^{2}+C s^{5} \iint_{\omega^{\prime} \times(0, T)} \mathrm{e}^{-8 s \alpha} \xi^{5}|\mathcal{D} \sigma|^{2} \mathrm{~d} x \mathrm{~d} t
$$


for every $s \geq C$. Finally, we are going to estimate the local term in (3.22) in terms of $g^{\varphi}, g^{\psi}, g^{\phi}, g^{\sigma}$ and a local term of $\phi$.

We recall the expression (3.14) for $\mathcal{D} \sigma$ valid in $\mathcal{O} \times(0, T)$ and consider a cut-off function $\zeta \in \mathcal{C}_{0}^{8}(\omega \cap \mathcal{O})$ such that $\zeta \equiv 1$ in $\omega^{\prime}$. Recall that $\omega_{0} \Subset \omega^{\prime} \Subset \omega \cap \mathcal{O}$. We have the following for the last term in (3.22):

$$
\begin{aligned}
& s^{5} \iint_{\omega^{\prime} \times(0, T)} \mathrm{e}^{-8 s \alpha} \xi^{5}|\mathcal{D} \sigma|^{2} \mathrm{~d} x \mathrm{~d} t \leq s^{5} \quad \iint_{(\omega \cap \mathcal{O}) \times(0, T)} \zeta(x) \mathrm{e}^{-8 s \alpha} \xi^{5}|\mathcal{D} \sigma|^{2} \mathrm{~d} x \mathrm{~d} t \\
= & -s^{5} \iiint_{(\omega \cap \mathcal{O}) \times(0, T)} \zeta(x) \mathrm{e}^{-8 s \alpha} \xi^{5} \mathcal{D} \sigma\left(\left(\Delta g_{3}^{\varphi}\right)_{t}-\Delta^{2} g_{3}^{\varphi}-\partial_{3} \nabla \cdot g_{t}^{\varphi}+\Delta\left(\partial_{3} \nabla \cdot g^{\varphi}\right)\right) \mathrm{d} x \mathrm{~d} t \\
& -s^{5} \iiint_{(\omega \cap \mathcal{O}) \times(0, T)} \zeta(x) \mathrm{e}^{-8 s \alpha} \xi^{5} \mathcal{D} \sigma\left(\Delta g_{3}^{\psi}-\partial_{3} \nabla \cdot g^{\psi}\right) \mathrm{d} x \mathrm{~d} t-s^{5} \iiint_{(\omega \cap \mathcal{O}) \times(0, T)} \zeta(x) \mathrm{e}^{-8 s \alpha} \xi^{5} \mathcal{D} \sigma \mathcal{P} g^{\phi} \mathrm{d} x \mathrm{~d} t \\
+ & s^{5} \underset{(\omega \cap \mathcal{O}) \times(0, T)}{\iint_{(\omega \cap \mathcal{O}) \times(0, T)} \zeta(x) \mathrm{e}^{-8 s \alpha} \xi^{5} \mathcal{D} \sigma\left(\Delta g_{t}^{\sigma}+\Delta^{2} g^{\sigma}\right) \mathrm{d} x \mathrm{~d} t-s^{5} \iint_{(x)} \zeta s \alpha} \mathrm{e}^{-8 s} \mathcal{D} \sigma\left[\mathcal{P}_{t}+\Delta \mathcal{P}\right] \phi \mathrm{d} x \mathrm{~d} t .
\end{aligned}
$$

Similarly as in Subsections 3.1.3 and 3.2, we need to integrate by parts in both time and space, keeping in mind the definition of the operator $\mathcal{P}$ (see (3.14)). Let us call the five integrals in (3.23) $A_{1}, \ldots, A_{5}$, respectively. A careful analysis of each of these terms (after integration by parts), taking into account properties (2.2), (3.3), (3.4) and Young's inequality, we can prove the following estimates for every $s \geq C$ :

$$
\begin{aligned}
& \left|A_{1}\right| \leq \frac{1}{8 C} J(\sigma)+C s^{15} \iint_{Q} \mathrm{e}^{-16 s \alpha+8 s \alpha^{*}} \xi^{18}\left|g^{\varphi}\right|^{2} \mathrm{~d} x \mathrm{~d} t \\
& \left|A_{2}\right| \leq \frac{1}{2 C} s^{5} \iint_{Q} \mathrm{e}^{8 s \alpha} \xi^{5}|\mathcal{D} \sigma|^{2} \mathrm{~d} x \mathrm{~d} t+C\left\|\mathrm{e}^{-7 / 2 s \alpha^{*}} g^{\psi}\right\|_{Y_{1}}^{2}, \\
& \left|A_{3}\right| \leq \frac{1}{8 C} J(\sigma)+C s^{19} \iint_{Q} \mathrm{e}^{-16 s \alpha+8 s \alpha^{*}} \xi^{22}\left|g^{\phi}\right|^{2} \mathrm{~d} x \mathrm{~d} t \\
& \left|A_{4}\right| \leq \frac{1}{8 C} J(\sigma)+C\left\|\mathrm{e}^{-7 / 2 s \alpha^{*}} g^{\sigma}\right\|_{X_{0}}^{2}, \\
& \left|A_{5}\right| \leq \frac{1}{8 C} J(\sigma)+C s^{23} \iint_{\omega \times(0, T)} \mathrm{e}^{-16 s \alpha+8 s \alpha^{*}} \xi^{26}|\phi|^{2} \mathrm{~d} x \mathrm{~d} t .
\end{aligned}
$$

From (3.22)-(3.28) we obtain

$$
\begin{aligned}
I_{4}(\sigma) \leq & C\left(\left\|s^{15 / 2} \mathrm{e}^{-8 s \alpha+4 s \alpha^{*}} \xi^{9} g^{\varphi}\right\|_{Y_{0}}^{2}+\left\|\mathrm{e}^{-7 / 2 s \alpha^{*}} g^{\psi}\right\|_{Y_{1}}^{2}+\left\|s^{19 / 2} \mathrm{e}^{-8 s \alpha+4 s \alpha^{*}} \xi^{11} g^{\phi}\right\|_{X_{0}}^{2}\right. \\
& \left.+\left\|\mathrm{e}^{-7 / 2 s \alpha^{*}} g^{\sigma}\right\|_{X_{4}}^{2}\right)+C s^{23} \iint_{\omega \times(0, T)} \mathrm{e}^{-16 s \alpha+8 s \alpha^{*}} \xi^{26}|\phi|^{2} \mathrm{~d} x \mathrm{~d} t,
\end{aligned}
$$

for every $s \geq C$. 


\subsubsection{Conclusion of the proof of (3.2)}

To conclude the proof of Proposition 3.1, we go back to (3.13), which combined with (3.29), taking into account that

$$
\begin{gathered}
s^{9} \mathrm{e}^{-9 s \alpha} \xi^{9} \leq C s^{15} \mathrm{e}^{-16 s \alpha+8 s \alpha^{*}} \xi^{18}, \\
s^{13} \mathrm{e}^{-9 s \alpha} \xi^{13} \leq C s^{19} \mathrm{e}^{-16 s \alpha+8 s \alpha^{*}} \xi^{22}, \\
s^{23} \mathrm{e}^{-16 s \alpha+8 s \alpha^{*}} \xi^{26} \leq C s^{26} \mathrm{e}^{-18 s \alpha+11 s \alpha^{*}} \xi^{30},
\end{gathered}
$$

for every $s \geq C$ and choosing $\varepsilon$ small enough we finally obtain

$$
\begin{aligned}
I_{1}(\varphi)+I_{2}(\psi)+I_{3}(\phi)+I_{4}(\sigma) \leq & C\left(\left\|s^{15 / 2} \mathrm{e}^{-8 s \alpha+4 s \alpha^{*}} \xi^{9} g^{\varphi}\right\|_{Y_{0}}^{2}+\left\|\mathrm{e}^{-7 / 2 s \alpha^{*}} g^{\psi}\right\|_{Y_{2}}^{2}\right. \\
& \left.+\left\|s^{19 / 2} \mathrm{e}^{-8 s \alpha+4 s \alpha^{*}} \xi^{11} g^{\phi}\right\|_{X_{0}}^{2}+\left\|\mathrm{e}^{-7 / 2 s \alpha^{*}} g^{\sigma}\right\|_{X_{4}}^{2}\right) \\
& +C s^{13} \iint_{\omega \times(0, T)} \mathrm{e}^{-9 s \alpha} \xi^{13}\left|\varphi_{1}\right|^{2} \mathrm{~d} x \mathrm{~d} t+C s^{26} \iint_{\omega \times(0, T)} \mathrm{e}^{-18 s \alpha+11 s \alpha^{*}} \xi^{30}|\phi|^{2} \mathrm{~d} x \mathrm{~d} t,
\end{aligned}
$$

for every $s \geq C$, from which we readily deduce (3.2).

This concludes the proof of Proposition 3.1.

\section{Null CONTROllability of The LineAR SYSTEM}

In this section we deal with the null controllability of system

$$
\begin{cases}\mathcal{L} w+\nabla p_{0}=f^{w}+v \mathbb{1}_{\omega}+r e_{N}, \nabla \cdot w=0 & \text { in } \quad Q, \\ \mathcal{L}^{*} z+\nabla p_{1}=f^{z}+w \mathbb{1}_{\mathcal{O}}, \nabla \cdot z=0 & \text { in } \quad Q, \\ \mathcal{L} r=f^{r}+v_{0} \mathbb{1}_{\omega} & \text { in } \quad Q, \\ \mathcal{L}^{*} q=f^{q}+z_{N}+r \mathbb{1}_{\mathcal{O}} & \text { in } \quad Q, \\ w=z=0, r=q=0 & \text { on } \quad \Sigma, \\ w(0)=0, z(T)=0, r(0)=0, q(T)=0 & \text { in } \Omega .\end{cases}
$$

Here, we will assume that $f^{w}, f^{z}, f^{r}$ and $f^{q}$ are in appropriate weighted functional spaces. We look for controls $\left(v, v_{0}\right)$, such that $v_{i_{0}} \equiv v_{N} \equiv 0$, for some given $0<i_{0}<N$, such that the associated solution of (4.1) satisfies $z(0)=0$ and $q(0)=0$ in $\Omega$.

To do this, let us first state a Carleman inequality with weight functions not vanishing in $t=T$. We introduce the following weight functions:

$$
\begin{aligned}
\beta(x, t) & =\frac{\mathrm{e}^{2 \lambda\|\eta\|_{\infty}}-\mathrm{e}^{\lambda \eta(x)}}{\widetilde{\ell}(t)^{m}}, \quad \gamma(x, t)=\frac{\mathrm{e}^{\lambda \eta(x)}}{\widetilde{\ell}(t)^{m}}, \\
\beta^{*}(t) & =\max _{x \in \bar{\Omega}} \alpha(x, t), \quad \gamma^{*}(t)=\min _{x \in \bar{\Omega}} \gamma(x, t), \\
\widehat{\beta}(t) & =\min _{x \in \bar{\Omega}} \beta(x, t), \quad \widehat{\gamma}(t)=\max _{x \in \bar{\Omega}} \gamma(x, t),
\end{aligned}
$$

where

$$
\widetilde{\ell}(t)= \begin{cases}\ell(t) & 0 \leq t \leq T / 2 \\ \|\ell\|_{\infty} & T / 2<t \leq T\end{cases}
$$


Lemma 4.1. Let $0<j_{0}<N$ and let $g^{\varphi}, g^{\phi}, s$ and $\lambda$ be like in Proposition 3.1. Furthermore, assume that $g^{\psi} \in$ $Y_{2,0}$ and $g^{\sigma} \in X_{4,0}$. Then, there exists a constant $C>0$ (depending on $s$ and $\lambda$ ) such that every solution $(\varphi, \psi, \phi, \sigma)$ of (3.1) satisfies

$$
\begin{aligned}
& \iint_{Q} \mathrm{e}^{-25 / 2 s \beta^{*}}\left(|\varphi|^{2}+|\psi|^{2}+|\phi|^{2}+|\sigma|^{2}\right) \mathrm{d} x \mathrm{~d} t \leq C\left(\left\|\mathrm{e}^{-13 / 4 s \beta^{*}} g^{\varphi}\right\|_{Y_{0}}^{2}\right. \\
& +\left\|\mathrm{e}^{-13 / 4 s \beta^{*}} g^{\psi}\right\|_{Y_{2,0}}^{2}+\left\|\mathrm{e}^{-13 / 4 s \beta^{*}} g^{\phi}\right\|_{X_{0}}^{2}+\left\|\mathrm{e}^{-13 / 4 s \beta^{*}} g^{\sigma}\right\|_{X_{4,0}}^{2} \\
& \left.+(N-2) \iint_{\omega \times(0, T)} \mathrm{e}^{-13 / 2 s \beta^{*}}\left|\varphi_{j_{0}}\right|^{2} \mathrm{~d} x \mathrm{~d} t+\iint_{\omega \times(0, T)} \mathrm{e}^{-13 / 2 s \beta^{*}}|\phi|^{2} \mathrm{~d} x \mathrm{~d} t\right) .
\end{aligned}
$$

To prove estimate (4.2) it suffices to combine (3.2) and classical energy estimates for the Stokes system and the heat equation satisfied by $\varphi, \psi, \phi$ and $\sigma$. For simplicity, we omit the proof. For more details on how to obtain $(4.2)$, see $[3,4]$ or [12]. Notice that, in order to obtain this more compact form, we have strongly used the property (3.3) and the assumptions $g^{\psi} \in Y_{2,0}$ and $g^{\sigma} \in X_{4,0}$ (see Sect. 2.1).

Remark 4.2. Observe that the additional assumptions on $g^{\psi}$ and $g^{\sigma}$ are not needed to obtain the energy estimates, but the fact that $\varphi(T) \equiv 0$ and $\phi(T) \equiv 0$ is essential.

Now we are ready to prove the null controllability of system (4.1). The idea is to look for a solution in an appropriate weighted functional space. To this end, we introduce, for $0<i_{0}<N$, the spaces

$$
\begin{aligned}
E_{i_{0}, s, \lambda}= & \left\{\left(w, p_{0}, z, p_{1}, r, q, v, v_{0}\right): \mathrm{e}^{13 / 4 s \beta^{*}} w \in L^{2}(Q)^{N}, \mathrm{e}^{13 / 4 s \beta^{*}} r \in L^{2}(Q),\right. \\
& \mathrm{e}^{13 / 4 s \beta^{*}} v \mathbb{1}_{\omega} \in L^{2}(Q)^{N}, v_{i_{0}} \equiv v_{N} \equiv 0, \mathrm{e}^{13 / 4 s \beta^{*}} v_{0} \mathbb{1}_{\omega} \in L^{2}(Q), \\
& \mathrm{e}^{13 / 4 s \beta^{*}}\left(\gamma^{*}\right)^{-1-1 / m} w \in Y_{1}, \mathrm{e}^{13 / 4 s \beta^{*}}\left(\gamma^{*}\right)^{-6-6 / m} z \in Y_{1}, z(T)=0, \\
& \mathrm{e}^{13 / 4 s \beta^{*}}\left(\gamma^{*}\right)^{-1-1 / m} r \in X_{1}, \mathrm{e}^{13 / 4 s \beta^{*}}\left(\gamma^{*}\right)^{-15-15 / m} q \in X_{1}, q(T)=0, \\
& \mathrm{e}^{25 / 4 s \beta^{*}}\left(\mathcal{L} w+\nabla p_{0}-v \mathbb{1}_{\omega}-r e_{N}, \mathcal{L}^{*} z+\nabla p_{1}-w \mathbb{1}_{\mathcal{O}}\right) \in L^{2}(Q)^{2 N}, \\
& \left.\mathrm{e}^{25 / 4 s \beta^{*}}\left(\mathcal{L} r-v_{0} \mathbb{1}_{\omega}, \mathcal{L}^{*} q-z_{N}-r \mathbb{1}_{\mathcal{O}}\right) \in L^{2}(Q)^{2}\right\} .
\end{aligned}
$$

It is clear that $E_{i_{0}, s, \lambda}$ is a Banach space endowed with its natural norm.

Remark 4.3. In particular, an element $\left(w, p_{0}, z, p_{1}, r, q, v, v_{0}\right) \in E_{i_{0}, s, \lambda}$ satisfies $w(0)=0, z(0)=$ $0, r(0)=0, q(0)=0, v_{i_{0}} \equiv v_{N} \equiv 0$. Moreover, since

$$
\mathrm{e}^{-a s \beta^{*}}\left(\gamma^{*}\right)^{c} \text { is bounded }
$$

for any $a>0$ and $c \in \mathbb{R}$, we have that

$$
\mathrm{e}^{25 / 4 s \beta^{*}}\left((w \cdot \nabla) w,(w \cdot \nabla) z,\left(z \cdot \nabla^{t}\right) w, q \nabla r, w \cdot \nabla r, w \cdot \nabla q\right) \in L^{2}(Q)^{4 N+2} .
$$

All the details are given in Section 5 .

Proposition 4.4. Assume the hypothesis of Lemma 4.1 and

$$
\mathrm{e}^{25 / 4 s \beta^{*}}\left(f^{w}, f^{z}, f^{r}, f^{q}\right) \in L^{2}(Q)^{2 N+2} .
$$

Let also $i_{0} \in\{1, \ldots, N-1\}$. Then, we can find controls $\left(v, v_{0}\right) \in L^{2}(Q)^{N+1}$ such that the associated solution $\left(w, p_{0}, z, p_{1}, r, q, v, v_{0}\right)$ to (4.1) belongs to $E_{i_{0}, s, \lambda}$. In particular, $v_{i_{0}} \equiv v_{N} \equiv 0$ and $(z(0), q(0))=(0,0)$ in $\Omega$. 
Proof. Following the arguments in [10,13], we introduce the space $P_{0}$ of functions $\left(\varphi, \pi_{\varphi}, \psi, \pi_{\psi}, \phi, \sigma\right) \in$ $\mathcal{C}^{\infty}(\bar{Q})^{2 N+4}$ such that

- $\nabla \cdot \varphi=\nabla \cdot \psi=0$,

- $\varphi_{\mid \Sigma}=\psi_{\mid \Sigma}=0, \phi_{\mid \Sigma}=\sigma_{\mid \Sigma}=0$,

- $\varphi(T)=\psi(0)=0, \phi(T)=\sigma(0)=0$,

- $\int_{\Omega} \pi_{\varphi} d x=\int_{\Omega} \pi_{\psi} d x=0$

- $\nabla \cdot\left(\mathcal{L} \psi+\nabla \pi_{\psi}-\sigma e_{N}\right)=0$,

- $\left(\mathcal{L}_{H}^{k}\left[\mathrm{e}^{-13 / 4 s \beta^{*}}\left(\mathcal{L} \psi+\nabla \pi_{\psi}-\sigma e_{N}\right)\right]\right)_{\mid \Sigma}=0, k=0,1$,

- $\left(\mathcal{L}_{H}^{k}\left[\mathrm{e}^{-13 / 4 s \beta^{*}}\left(\mathcal{L} \psi+\nabla \pi_{\psi}-\sigma e_{N}\right)\right]\right)(0)=0, k=0,1$,

- $\mathcal{L}^{k}\left[\mathrm{e}^{-13 / 4 s \beta^{*}} \mathcal{L} \sigma\right]_{\mid \Sigma}=0, k=0, \ldots, 3$,

- $\mathcal{L}^{k}\left[\mathrm{e}^{-13 / 4 s \beta^{*}} \mathcal{L} \sigma\right](0)=0, k=0, \ldots, 3$.

We define the bilinear form

$$
\begin{aligned}
& a\left(\left(\widetilde{\varphi}, \widetilde{\pi}_{\varphi}, \widetilde{\psi}, \widetilde{\pi}_{\psi}, \widetilde{\phi}, \widetilde{\sigma}\right),\left(\varphi, \pi_{\varphi}, \psi, \pi_{\psi}, \phi, \sigma\right)\right) \\
:= & \iint_{Q} \mathrm{e}^{-13 / 2 s \beta^{*}}\left(\mathcal{L}^{*} \widetilde{\varphi}+\nabla \widetilde{\pi}_{\varphi}-\widetilde{\psi} \mathbb{1}_{\mathcal{O}}\right) \cdot\left(\mathcal{L}^{*} \varphi+\nabla \pi_{\varphi}-\psi \mathbb{1}_{\mathcal{O}}\right) \mathrm{d} x \mathrm{~d} t \\
& +\iint_{Q} \mathcal{L}_{H}^{2}\left[\mathrm{e}^{-13 / 4 s \beta^{*}}\left(\mathcal{L} \widetilde{\psi}+\nabla \widetilde{\pi}_{\psi}-\widetilde{\sigma} e_{N}\right)\right] \cdot \mathcal{L}_{H}^{2}\left[\mathrm{e}^{-13 / 4 s \beta^{*}}\left(\mathcal{L} \psi+\nabla \pi_{\psi}-\sigma e_{N}\right)\right] \mathrm{d} x \mathrm{~d} t \\
& +\iint_{Q} \mathrm{e}^{-13 / 2 s \beta^{*}}\left(\mathcal{L}^{*} \widetilde{\phi}-\widetilde{\varphi}_{N}-\widetilde{\sigma} \mathbb{1}_{\mathcal{O}}\right)\left(\mathcal{L}^{*} \phi-\varphi_{N}-\sigma \mathbb{1}_{\mathcal{O}}\right) \mathrm{d} x \mathrm{~d} t \\
& +\iint_{Q} \mathcal{L}^{4}\left[\mathrm{e}^{-13 / 4 s \beta^{*}} \mathcal{L} \widetilde{\sigma}\right] \mathcal{L}^{4}\left[\mathrm{e}^{-13 / 4 s \beta^{*}} \mathcal{L} \sigma\right] \mathrm{d} x \mathrm{~d} t \\
& +(N-2) \iint_{\omega \times(0, T)} \mathrm{e}^{-13 / 2 s \beta^{*}} \widetilde{\varphi}_{j_{0}} \varphi_{j_{0}} \mathrm{~d} x \mathrm{~d} t+\iint_{\omega \times(0, T)} \mathrm{e}^{-13 / 2 s \beta^{*}} \widetilde{\phi} \phi \mathrm{d} x \mathrm{~d} t,
\end{aligned}
$$

where $j_{0} \in\{1, \ldots, N-1\} \backslash\left\{i_{0}\right\}$ and a linear form

$$
\left\langle G,\left(\varphi, \pi_{\varphi}, \psi, \pi_{\psi}, \phi, \sigma\right)\right\rangle:=\iint_{Q} f^{w} \cdot \varphi \mathrm{d} x \mathrm{~d} t+\iint_{Q} f^{z} \cdot \psi \mathrm{d} x \mathrm{~d} t+\iint_{Q} f^{r} \phi \mathrm{d} x \mathrm{~d} t+\iint_{Q} f^{q} \sigma \mathrm{d} x \mathrm{~d} t .
$$

Thanks to (4.2), we have that $a(\cdot, \cdot): P_{0} \times P_{0} \mapsto \mathbb{R}$ is a symmetric, definite positive bilinear form on $P_{0}$. We denote by $P$ the completion of $P_{0}$ for the norm induced by $a(\cdot, \cdot)$. Then, $a(\cdot, \cdot)$ is well-defined, continuous and definite positive on $P$. Furthermore, in view of the Carleman estimate (4.2) and the assumptions (4.4), the linear form $\left(\varphi, \pi_{\varphi}, \psi, \pi_{\psi}, \phi, \sigma\right) \mapsto\left\langle G,\left(\varphi, \pi_{\varphi}, \psi, \pi_{\psi}, \phi, \sigma\right)\right\rangle$ is well-defined and continuous on $P$. Hence, from Lax-Milgram's lemma, we deduce that the variational problem:

$$
\left\{\begin{array}{l}
\text { Find }\left(\widetilde{\varphi}, \tilde{\pi}_{\varphi}, \widetilde{\psi}, \widetilde{\pi}_{\psi}, \widetilde{\phi}, \widetilde{\sigma}\right) \in P \text { such that } \\
a\left(\left(\widetilde{\varphi}, \tilde{\pi}_{\varphi}, \widetilde{\psi}, \tilde{\pi}_{\psi}, \widetilde{\phi}, \widetilde{\sigma}\right),\left(\varphi, \pi_{\varphi}, \psi, \pi_{\psi}, \phi, \sigma\right)\right)=\left\langle G,\left(\varphi, \pi_{\varphi}, \psi, \pi_{\psi}, \phi, \sigma\right)\right\rangle \quad \forall\left(\varphi, \pi_{\varphi}, \psi, \pi_{\psi}, \phi, \sigma\right) \in P,
\end{array}\right.
$$

possesses exactly one solution $\left(\widehat{\varphi}, \widehat{\pi}_{\varphi}, \widehat{\psi}, \widehat{\pi}_{\psi}, \widehat{\phi}, \widehat{\sigma}\right)$.

Let $\widehat{v}$ and $\widehat{v}_{0}$ be given by

$$
\begin{cases}\widehat{v}_{j_{0}}:=-(N-2) \mathrm{e}^{-13 / 2 s \beta^{*}} \widehat{\varphi}_{j_{0}} \mathbb{1}_{\omega}, \widehat{v}_{j} \equiv 0, j \neq j_{0}, & \text { in } \quad Q, \\ \widehat{v}_{0}:=-\mathrm{e}^{-13 / 2 s \beta^{*}} \widehat{\phi}_{\omega}, & \text { in } Q .\end{cases}
$$


It is straightforward from (4.5) and (4.6) that we have

$$
\iint_{Q}\left(|\widetilde{w}|^{2}+|\widetilde{z}|^{2}+|\widetilde{r}|^{2}+|\widetilde{q}|^{2}\right) \mathrm{d} x \mathrm{~d} t+\iint_{\omega \times(0, T)} \mathrm{e}^{13 / 2 s \beta^{*}}\left((N-2)\left|\widehat{v}_{j_{0}}\right|^{2}+\left|\widehat{v}_{0}\right|^{2}\right) \mathrm{d} x \mathrm{~d} t<+\infty,
$$

where $\widetilde{w}, \widetilde{z}, \widetilde{r}$ and $\widetilde{q}$ are given by

$$
\left\{\begin{array}{l}
\widetilde{w}:=\mathrm{e}^{-13 / 4 s \beta^{*}}\left(\mathcal{L}^{*} \widehat{\varphi}+\nabla \widehat{\pi}_{\varphi}-\widehat{\psi} \mathbb{1}_{\mathcal{O}}\right), \\
\widetilde{z}:=\mathcal{L}_{H}^{2}\left[\mathrm{e}^{-13 / 4 s \beta^{*}}\left(\mathcal{L} \widehat{\psi}+\nabla \widehat{\pi}_{\psi}-\widehat{\sigma} e_{N}\right)\right], \\
\widetilde{r}:=\mathrm{e}^{-13 / 4 s \beta^{*}}\left(\mathcal{L}^{*} \widehat{\phi}-\widehat{\varphi}_{N}-\widehat{\sigma} \mathbb{1}_{\mathcal{O}}\right), \\
\widetilde{q}:=\mathcal{L}^{4}\left[\mathrm{e}^{-13 / 4 s \beta^{*}} \mathcal{L} \widehat{\sigma}\right] .
\end{array}\right.
$$

In particular, $\widehat{v} \in L^{2}(Q)^{N}, \widehat{v}_{0} \in L^{2}(Q)$.

Let us call $(\widehat{w}, \widehat{z}, \widehat{r}, \widehat{q})$, together with some pressures $\left(\widehat{p}_{0}, \widehat{p}_{1}\right)$, the (weak) solution of (4.1) with $v=\widehat{v}$ and $v_{0}=\widehat{v}_{0}$, that is, they solve

$$
\begin{cases}\mathcal{L} \widehat{w}+\nabla \widehat{p}_{0}=f^{w}+\widehat{v} \mathbb{1}_{\omega}+\widehat{r} e_{N}, \nabla \cdot \widehat{w}=0 & \text { in } \quad Q, \\ \mathcal{L}^{*} \widehat{z}+\nabla \widehat{p}_{1}=f^{z}+\widehat{w} \mathbb{1}_{\mathcal{O}}, \nabla \cdot \widehat{z}=0 & \text { in } \quad Q, \\ \mathcal{L} \widehat{r}=f^{r}+\widehat{v}_{0} \mathbb{1}_{\omega} & \text { in } \quad Q, \\ \mathcal{L}^{*} \widehat{q}=f^{q}+\widehat{z}_{N}+\widehat{r} \mathbb{1}_{\mathcal{O}} & \text { in } \quad Q, \\ \widehat{w}=\widehat{z}=0, \widehat{r}=\widehat{q}=0 & \text { on } \quad \Sigma, \\ \widehat{w}(0)=0, \widehat{z}(T)=0, \widehat{r}(0)=0, \widehat{q}(T)=0 & \text { in } \quad \Omega .\end{cases}
$$

The rest of the proof is devoted to prove the following exponential decay properties

$$
\begin{aligned}
& \mathrm{e}^{13 / 4 s \beta^{*}}\left(\gamma^{*}\right)^{-1-1 / m} \widehat{w} \in Y_{1}, \quad \mathrm{e}^{13 / 4 s \beta^{*}}\left(\gamma^{*}\right)^{-6-6 / m} \widehat{z} \in Y_{1} \\
& \mathrm{e}^{13 / 4 s \beta^{*}}\left(\gamma^{*}\right)^{-1-1 / m} \widehat{r} \in X_{1}, \quad \mathrm{e}^{13 / 4 s \beta^{*}}\left(\gamma^{*}\right)^{-15-15 / m} \widehat{q} \in X_{1},
\end{aligned}
$$

which will solve the null controllability problem for system (4.1) (see Rem. 4.3).

First, we will prove that $(\widetilde{w}, \widetilde{z}, \widetilde{r}, \widetilde{q})$ given by (4.8) is actually the solution (in the sense of transposition) of

$$
\begin{cases}\mathrm{e}^{-13 / 4 s \beta^{*}} \widetilde{w}=\widehat{w} & \text { in } \quad Q, \\ \mathrm{e}^{-13 / 4 s \beta^{*}}\left(\mathcal{L}_{H}^{*}\right)^{2} \widetilde{z}=\widehat{z}, \nabla \cdot \widetilde{z}=0 & \text { in } Q, \\ \mathrm{e}^{-13 / 4 s \beta^{*}} \widetilde{r}=\widehat{r} & \text { in } Q, \\ \mathrm{e}^{-13 / 4 s \beta^{*}}\left(\mathcal{L}^{*}\right)^{4} \widetilde{q}=\widehat{q} & \text { in } \quad Q,\end{cases}
$$

such that

$$
\begin{cases}\left(\mathcal{L}_{H}^{*}\right)^{\ell} \widetilde{z}=0 & \text { on } \quad \Sigma, \quad \ell=0,1, \\ \left(\mathcal{L}_{H}^{*}\right)^{\ell} \widetilde{z}(T)=0 & \text { in } \quad \Omega, \quad \ell=0,1, \\ \left(\mathcal{L}^{*}\right)^{k} \widetilde{q}=0 & \text { on } \quad \Sigma, \quad k=0, \ldots, 3, \\ \left(\mathcal{L}^{*}\right)^{k} \widetilde{q}(T)=0 & \text { in } \quad \Omega, \quad k=0, \ldots, 3 .\end{cases}
$$

Now, from (4.5), (4.6), (4.8) and (4.9), we obtain for every $\left(\varphi, \pi_{\varphi}, \psi, \pi_{\psi}, \phi, \sigma\right) \in P_{0}$

$$
\begin{aligned}
& \iint_{Q} \widetilde{w} \cdot \mathrm{e}^{-13 / 4 s \beta^{*}}\left(\mathcal{L}^{*} \varphi+\nabla \pi_{\varphi}-\psi \mathbb{1}_{\mathcal{O}}\right) \mathrm{d} x \mathrm{~d} t+\iint_{Q} \widetilde{z} \cdot \mathcal{L}_{H}^{2}\left[\mathrm{e}^{-13 / 4 s \beta^{*}}\left(\mathcal{L} \psi+\nabla \pi_{\psi}-\sigma e_{N}\right)\right] \mathrm{d} x \mathrm{~d} t \\
& +\iint_{Q} \widetilde{r} \mathrm{e}^{-13 / 4 s \beta^{*}}\left(\mathcal{L}^{*} \phi-\varphi_{N}-\sigma \mathbb{1}_{\mathcal{O}}\right) \mathrm{d} x \mathrm{~d} t+\iint_{Q} \widetilde{q} \mathcal{L}^{4}\left[\mathrm{e}^{-13 / 4 s \beta^{*}} \mathcal{L} \sigma\right] \mathrm{d} x \mathrm{~d} t
\end{aligned}
$$




$$
\begin{aligned}
& =\iint_{Q} \varphi \cdot\left(\mathcal{L} \widehat{w}+\nabla \widehat{p}_{0}-\widehat{r} e_{N}\right) \mathrm{d} x \mathrm{~d} t+\iint_{Q} \psi \cdot\left(\mathcal{L}^{*} \widehat{z}+\nabla \widehat{p}_{1}-\widehat{w} \mathbb{1}_{\mathcal{O}}\right) \mathrm{d} x \mathrm{~d} t \\
& \quad+\iint_{Q} \phi \mathcal{L} \widehat{r} \mathrm{~d} x \mathrm{~d} t+\iint_{Q} \sigma\left(\mathcal{L}^{*} \widehat{q}-\widehat{z}_{N}-\widehat{r} \mathbb{1}_{\mathcal{O}}\right) \mathrm{d} x \mathrm{~d} t \\
& =\iint_{Q} \widehat{w} \cdot\left(\mathcal{L}^{*} \varphi+\nabla \pi_{\varphi}-\psi \mathbb{1}_{\mathcal{O}}\right) \mathrm{d} x \mathrm{~d} t+\iint_{Q} \widehat{z} \cdot\left(\mathcal{L} \psi+\nabla \pi_{\psi}-\sigma e_{N}\right) \mathrm{d} x \mathrm{~d} t \\
& \quad+\iint_{Q} \widehat{r}\left(\mathcal{L}^{*} \phi-\varphi_{N}-\sigma \mathbb{1}_{\mathcal{O}}\right) \mathrm{d} x \mathrm{~d} t+\iint_{Q} \widehat{q} \mathcal{L} \sigma \mathrm{d} x \mathrm{~d} t .
\end{aligned}
$$

From this last equality, we obtain for all $\left(h^{w}, h^{z}, h^{r}, h^{q}\right) \in L^{2}(Q)^{2 N+2}$

$$
\begin{aligned}
& \iint_{Q} \widetilde{w} \cdot h^{w} \mathrm{~d} x \mathrm{~d} t+\iint_{Q} \widetilde{z} \cdot h^{z} \mathrm{~d} x \mathrm{~d} t+\iint_{Q} \widetilde{r} h^{r} \mathrm{~d} x \mathrm{~d} t+\iint_{Q} \widetilde{q} h^{q} \mathrm{~d} x \mathrm{~d} t \\
& =\iint_{Q} \widehat{w} \cdot \Phi^{w} \mathrm{~d} x \mathrm{~d} t+\iint_{Q} \widehat{z} \cdot \Phi^{z} \mathrm{~d} x \mathrm{~d} t+\iint_{Q} \widehat{r} \Phi^{r} \mathrm{~d} x \mathrm{~d} t+\iint_{Q} \widehat{q} \Phi^{q} \mathrm{~d} x \mathrm{~d} t,
\end{aligned}
$$

where $\left(\Phi^{w}, \Phi^{z}, \Phi^{r}, \Phi^{q}\right)$ is the solution of

$$
\begin{cases}\mathrm{e}^{-13 / 4 s \beta^{*}} \Phi^{w}=h^{w} & \text { in } Q, \\ \mathcal{L}_{H}^{2}\left[\mathrm{e}^{-13 / 4 s \beta^{*}} \Phi^{z}\right]=h^{z}, \nabla \cdot \Phi^{z}=0 & \text { in } Q, \\ \mathrm{e}^{-13 / 4 s \beta^{*}} \Phi^{r}=h^{r} & \text { in } Q, \\ \mathcal{L}^{4}\left[\mathrm{e}^{-13 / 4 s \beta^{*}} \Phi^{q}\right]=h^{q} & \text { in } Q,\end{cases}
$$

such that

$$
\left\{\begin{array}{lll}
\mathcal{L}_{H}^{\ell}\left(\mathrm{e}^{-13 / 4 s \beta^{*}} \Phi^{z}\right)=0 & \text { on } \quad \Sigma, \quad \ell=0,1, \\
\mathcal{L}_{H}^{\ell}\left(\mathrm{e}^{-13 / 4 s \beta^{*}} \Phi^{z}\right)(0)=0 & \text { in } \quad \Omega, \quad \ell=0,1, \\
\mathcal{L}^{k}\left(\mathrm{e}^{-13 / 4 s \beta^{*}} \Phi^{q}\right)=0 & \text { on } \quad \Sigma, \quad k=0, \ldots, 3, \\
\mathcal{L}^{k}\left(\mathrm{e}^{-13 / 4 s \beta^{*}} \Phi^{q}\right)(0)=0 & \text { in } \quad \Omega, \quad k=0, \ldots, 3 .
\end{array}\right.
$$

It is classical to show that (4.13)-(4.15) is equivalent to (4.11) and (4.12).

Next, we define the following functions:

$$
\left(z_{*, 0}, p_{*, 0}\right):=\mathrm{e}^{13 / 4 s \beta^{*}}\left(\gamma^{*}\right)^{-3-3 / m}\left(\widehat{z}, \widehat{p}_{1}\right), \quad f_{*, 0}^{z}:=\mathrm{e}^{13 / 4 s \beta^{*}}\left(\gamma^{*}\right)^{-3-3 / m}\left(f^{z}+\widehat{w} \mathbb{1}_{\mathcal{O}}\right) .
$$

Observe that, from (4.4), (4.7) and (4.11), we have $f_{*, 0}^{z} \in L^{2}(Q)^{N}$. Then, by (4.9) $z_{*, 0}$ satisfies

$$
\begin{cases}\mathcal{L}^{*} z_{*, 0}+\nabla p_{*, 0}=f_{*, 0}^{z}-\left(\mathrm{e}^{13 / 4 s \beta^{*}}\left(\gamma^{*}\right)^{-3-3 / m}\right)_{t} \widehat{z}, \nabla \cdot z_{*, 0}=0 & \text { in } Q, \\ z_{*, 0}=0 & \text { on } \Sigma, \\ z_{*, 0}(T)=0 & \text { in } \Omega,\end{cases}
$$

where the last term in the right-hand side can be written as

$$
\left(\mathrm{e}^{13 / 4 s \beta^{*}}\left(\gamma^{*}\right)^{-3-3 / m}\right)_{t} \widehat{z}=c_{2}(t)\left(\mathcal{L}_{H}^{*}\right)^{2} \widetilde{z}
$$


where $c_{k}(t)$ denotes a generic function such that (see (2.2))

$$
\left|c_{k}^{(\ell)}(t)\right| \leq C<\infty, \quad \forall \ell=0, \ldots, k .
$$

On the other hand, for any $h \in Y_{1,0}$ we have

$$
\iint_{Q} z_{*, 0} \cdot h \mathrm{~d} x \mathrm{~d} t=\iint_{Q} f_{*, 0}^{z} \cdot \Phi \mathrm{d} x \mathrm{~d} t-\iint_{Q} c_{2}(t)\left(\mathcal{L}^{*}\right)_{H}^{2} \widetilde{z} \cdot \Phi \mathrm{d} x \mathrm{~d} t,
$$

where $\Phi$ solves, together with some pressure $\pi_{\Phi}$,

$$
\begin{cases}\mathcal{L} \Phi+\nabla \pi_{\Phi}=h, \quad \nabla \cdot \Phi=0 & \text { in } Q \\ \Phi=0 & \text { on } \Sigma \\ \Phi(0)=0 & \text { in } \Omega .\end{cases}
$$

Using (4.12), we can integrate by parts to obtain

$$
\begin{aligned}
\iint_{Q} z_{*, 0} \cdot h \mathrm{~d} x \mathrm{~d} t & =\iint_{Q} f_{*, 0}^{z} \cdot \Phi \mathrm{d} x \mathrm{~d} t-\iint_{Q} \mathcal{L}_{H}^{*} \widetilde{z} \cdot\left(\mathcal{L}\left[c_{2}(t) \Phi\right]+\nabla\left(c_{2}(t) \pi_{\Phi}\right)\right) \mathrm{d} x \mathrm{~d} t \\
& =\iint_{Q} f_{*, 0}^{z} \cdot \Phi \mathrm{d} x \mathrm{~d} t-\iint_{Q} \widetilde{z} \cdot \mathcal{L}\left[c_{2}^{\prime}(t) \Phi+c_{2}(t) h\right] \mathrm{d} x \mathrm{~d} t .
\end{aligned}
$$

Notice that here we have relied on the fact that $\mathcal{L}_{H}^{*} \widetilde{z}, \Phi$ and $h$ belong to the space $H$. Since

$$
\|\Phi\|_{Y_{2}} \leq C\|h\|_{Y_{1,0}}
$$

(see regularity result (2.9)), we obtain from the last equality, together with (4.16),

$$
\iint_{Q} z_{*, 0} \cdot h \mathrm{~d} x \mathrm{~d} t \leq C\left[\left\|f_{*, 0}^{z}\right\|_{L^{2}(Q)^{N}}+\|\widetilde{z}\|_{L^{2}(Q)^{N}}\right]\|h\|_{Y_{1,0}}, \quad \forall h \in Y_{1,0} .
$$

Now, let

$$
\left(z_{*, 1}, p_{*, 1}\right):=\mathrm{e}^{13 / 4 s \beta^{*}}\left(\gamma^{*}\right)^{-5-5 / m}\left(\widehat{z}, \widehat{p}_{1}\right), \quad f_{*, 1}^{z}:=\mathrm{e}^{13 / 4 s \beta^{*}}\left(\gamma^{*}\right)^{-5-5 / m}\left(f^{z}+\widehat{w} \mathbb{1}_{\mathcal{O}}\right) .
$$

Similarly as before, $\left(z_{*, 1}, p_{*, 1}\right)$ satisfies

$$
\begin{cases}\mathcal{L}^{*} z_{*, 1}+\nabla p_{*, 1}=f_{*, 1}^{z}-\left(\mathrm{e}^{13 / 4 s \beta^{*}}\left(\gamma^{*}\right)^{-5-5 / m}\right)_{t} \widehat{z}, \nabla \cdot z_{*, 1}=0 & \text { in } \quad Q, \\ z_{*, 1}=0 & \text { on } \quad \Sigma, \\ z_{*, 1}(T)=0 & \text { in } \quad \Omega .\end{cases}
$$

Thus, for any $h \in Y_{0}$ we get

$$
\iint_{Q} z_{*, 1} \cdot h \mathrm{~d} x \mathrm{~d} t=\iint_{Q} f_{*, 1}^{z} \cdot \Phi \mathrm{d} x \mathrm{~d} t-\iint_{Q}\left(\mathrm{e}^{13 / 4 s \beta^{*}}\left(\gamma^{*}\right)^{-5-5 / m}\right)_{t} \widehat{z} \cdot \Phi \mathrm{d} x \mathrm{~d} t .
$$

Moreover, since

$$
\iint_{Q}\left(\mathrm{e}^{13 / 4 s \beta^{*}}\left(\gamma^{*}\right)^{-5-5 / m}\right)_{t} \widehat{z} \cdot \Phi \mathrm{d} x \mathrm{~d} t=\iint_{Q} c_{1}(t) \Phi \cdot z_{*, 0} \mathrm{~d} x \mathrm{~d} t
$$


using (4.17) with $c_{1}(t) \Phi$ instead of $h$ (notice that $\left.c_{1}(t) \Phi \in Y_{1,0}\right)$, we get the estimate

$$
\iint_{Q} c_{1}(t) \Phi \cdot z_{*, 0} \mathrm{~d} x \mathrm{~d} t \leq C\left[\left\|f_{*, 0}^{z}\right\|_{L^{2}(Q)^{N}}+\|\widetilde{z}\|_{L^{2}(Q)^{N}}\right]\left\|c_{1}(t) \Phi\right\|_{Y_{1,0}} .
$$

Turning back to $z_{*, 1}$, we get

$$
\iint_{Q} z_{*, 1} \cdot h \mathrm{~d} x \mathrm{~d} t \leq C\left[\left\|f_{*, 0}^{z}\right\|_{L^{2}(Q)^{N}}+\|\widetilde{z}\|_{L^{2}(Q)^{N}}\right]\|\Phi\|_{Y_{1,0}}
$$

where we have used (4.16) and the property $\left(\gamma^{*}\right)^{-5-5 / m} \leq C\left(\gamma^{*}\right)^{-3-3 / m}$. Taking into account that

$$
\|\Phi\|_{Y_{1,0}} \leq C\|h\|_{Y_{0}}
$$

(see (2.8)) we obtain

$$
\iint_{Q} z_{*, 1} \cdot h \mathrm{~d} x \mathrm{~d} t \leq C\left[\left\|f_{*, 0}^{z}\right\|_{L^{2}(Q)^{N}}+\|\widetilde{z}\|_{L^{2}(Q)^{N}}\right]\|h\|_{Y_{0}}, \quad \forall h \in Y_{0} .
$$

Thus, we deduce that $z_{*, 1} \in L^{2}(Q)^{N}$. Following the same iterative argument we can show that $\mathrm{e}^{13 / 4 s \beta^{*}}\left(\gamma^{*}\right)^{-14-14 / m} \widehat{q} \in L^{2}(Q)^{N}$.

Finally, to complete the proof of (4.10), let

$$
\left(z_{*}, p_{1 *}\right):=\mathrm{e}^{13 / 4 s \beta^{*}}\left(\gamma^{*}\right)^{-6-6 / m}\left(\widehat{z}, \widehat{p}_{1}\right), \quad f_{*}^{z}:=\mathrm{e}^{13 / 4 s \beta^{*}}\left(\gamma^{*}\right)^{-6-6 / m}\left(f^{z}+\widehat{w} \mathbb{1}_{\mathcal{O}}\right) .
$$

Then, $\left(z_{*}, p_{1 *}\right)$ satisfies

$$
\begin{cases}\mathcal{L}^{*} z_{*}+\nabla p_{1 *}=f_{*}^{z}+\left(\mathrm{e}^{13 / 4 s \beta^{*}}\left(\gamma^{*}\right)^{-6-6 / m}\right)_{t} \widehat{z}, \nabla \cdot z_{*}=0 & \text { in } Q \\ z_{*}=0 & \text { on } \Sigma, \\ z_{*}(T)=0 & \text { in } \Omega .\end{cases}
$$

From (4.4), (4.7), (4.11), (2.2) and $z_{*, 1} \in L^{2}(Q)^{N}$, we have that the right-hand side of this equation belongs to $L^{2}(Q)^{N}$. Using the regularity result (2.8), we deduce that $z_{*} \in Y_{1}$. Similarly, we are able to obtain the rest of the regularity properties in (4.10). This concludes the proof of Proposition 4.4.

\section{Proof of Theorem 1.1}

Recall that we deal with the following null controllability problem: to find controls $\left(v, v_{0}\right)$ verifying $v_{i_{0}} \equiv$ $v_{N} \equiv 0$ such that the solution of the system

$$
\begin{cases}\mathcal{L} w+(w \cdot \nabla) w+\nabla p_{0}=f+v \mathbb{1}_{\omega}+r e_{N}, \nabla \cdot w=0 & \text { in } \quad Q, \\ \mathcal{L}^{*} z+\left(z \cdot \nabla^{t}\right) w-(w \cdot \nabla) z+q \nabla r+\nabla p_{1}=w \mathbb{1}_{\mathcal{O}}, \nabla \cdot z=0 & \text { in } Q, \\ \mathcal{L} r+w \cdot \nabla r=f_{0}+v_{0} \mathbb{1}_{\omega} & \text { in } \quad Q, \\ \mathcal{L}^{*} q-w \cdot \nabla q=z_{N}+r \mathbb{1}_{\mathcal{O}} & \text { in } \quad Q, \\ w=z=0, r=q=0 & \text { on } \Sigma, \\ w(0)=0, z(T)=0, r(0)=0, q(T)=0 & \text { in } \Omega,\end{cases}
$$

satisfies $(z(0), q(0))=(0,0)$.

We proceed using similar arguments to those in [13] (see also $[4,5,9,12]$ ). The null controllability result for the linear system given by Proposition 4.4 will allow us to apply the following inverse mapping theorem (see [1]): 
Theorem 5.1. Let $\mathcal{G}_{1}$ and $\mathcal{G}_{2}$ be two Banach spaces and let $\mathcal{F}: \mathcal{G}_{1} \rightarrow \mathcal{G}_{2}$ satisfy $\mathcal{F} \in \mathcal{C}^{1}\left(\mathcal{G}_{1} ; \mathcal{G}_{2}\right)$. Assume that $g_{1} \in \mathcal{G}_{1}, \mathcal{F}\left(g_{1}\right)=g_{2}$ and that $\mathcal{F}^{\prime}\left(g_{1}\right): \mathcal{G}_{1} \rightarrow \mathcal{G}_{2}$ is surjective. Then, there exists $\delta>0$ such that, for every $g^{\prime} \in \mathcal{G}_{2}$ satisfying $\left\|g^{\prime}-g_{2}\right\|_{\mathcal{G}_{2}}<\delta$, there exists a solution of the equation

$$
\mathcal{F}(g)=g^{\prime}, \quad g \in \mathcal{G}_{1} .
$$

Let us set the framework to apply Theorem 5.1 to the problem at hand. Let

$$
\begin{gathered}
\mathcal{G}_{1}:=E_{i_{0}, s, \lambda}, \\
\mathcal{G}_{2}:=L^{2}\left(\mathrm{e}^{25 / 4 s \beta^{*}}(0, T) ; L^{2}(\Omega)^{2 N+2}\right)
\end{gathered}
$$

and the operator

$$
\begin{aligned}
\mathcal{F}\left(w, p_{0}, z, p_{1}, r, q, v, v_{0}\right):= & \left(\mathcal{L} w+(w \cdot \nabla) w+\nabla p_{0}-v \mathbb{1}_{\omega}-r e_{N},\right. \\
& \mathcal{L}^{*} z+\left(z \cdot \nabla^{t}\right) w-(w \cdot \nabla) z+q \nabla r+\nabla p_{1}-w \mathbb{1}_{\mathcal{O}}, \\
& \left.\mathcal{L} r+w \cdot \nabla r-v_{0} \mathbb{1}_{\omega}, \mathcal{L}^{*} q-w \cdot \nabla q-z_{N}-r \mathbb{1}_{\mathcal{O}}\right)
\end{aligned}
$$

for $\left(w, p_{0}, z, p_{1}, r, q, v, v_{0}\right) \in \mathcal{G}_{1}$. Here, $u \in L^{2}\left(\mathrm{e}^{25 / 4 s \beta^{*}}(0, T) ; L^{2}(\Omega)\right)$ means $\mathrm{e}^{25 / 4 s \beta^{*}} u \in L^{2}(Q)$.

It only remains to check that the operator $\mathcal{F}$ is of class $\mathcal{C}^{1}\left(\mathcal{G}_{1} ; \mathcal{G}_{2}\right)$. To do this, we notice that all the terms in $\mathcal{F}$ are linear, except for $(w \cdot \nabla) w,\left(z \cdot \nabla^{t}\right) w-(w \cdot \nabla) z, q \nabla r, w \cdot \nabla r$ and $w \cdot \nabla q$. So it will suffice to prove that the bilinear operator

$$
\left(\left(w^{1}, p_{0}^{1}, z^{1}, p_{1}^{1}, r^{1}, q^{1}, v^{1}, v_{0}^{1}\right),\left(w^{2}, p_{0}^{2}, z^{2}, p_{1}^{2}, r^{2}, q^{2}, v^{2}, v_{0}^{2}\right)\right) \rightarrow\left(w^{1} \cdot \nabla\right) w^{2}
$$

is continuous from $\mathcal{G}_{1} \times \mathcal{G}_{1}$ to $L^{2}\left(\mathrm{e}^{25 / 4 s \beta^{*}}(0, T) ; L^{2}(\Omega)^{N}\right)$. Since $Y_{1} \subset L^{\infty}(0, T ; V)$, we have that

$$
\mathrm{e}^{13 / 4 s \beta^{*}}\left(\gamma^{*}\right)^{-1-1 / m} w \in L^{2}\left(0, T ; H^{2}(\Omega)^{N}\right) \cap L^{\infty}(0, T ; V)
$$

for any $\left(w, p_{0}, z, p_{1}, r, q, v, v_{0}\right) \in \mathcal{G}_{1}$. Consequently

$$
\mathrm{e}^{13 / 4 s \beta^{*}}\left(\gamma^{*}\right)^{-1-1 / m} w \in L^{2}\left(0, T ; L^{\infty}(\Omega)^{N}\right)
$$

and

$$
\nabla\left(\mathrm{e}^{13 / 4 s \beta^{*}}\left(\gamma^{*}\right)^{-1-1 / m} w\right) \in L^{\infty}\left(0, T ; L^{2}(\Omega)^{N \times N}\right)
$$

Thus, we obtain

$$
\begin{aligned}
& \left\|\mathrm{e}^{13 / 2 s \beta^{*}}\left(\gamma^{*}\right)^{-2-2 / m}\left(w^{1} \cdot \nabla\right) w^{2}\right\|_{L^{2}(Q)^{N}} \\
& \leq C\left\|\left(\mathrm{e}^{13 / 4 s \beta^{*}}\left(\gamma^{*}\right)^{-1-1 / m} w^{1} \cdot \nabla\right) \mathrm{e}^{13 / 4 s \beta^{*}}\left(\gamma^{*}\right)^{-1-1 / m} w^{2}\right\|_{L^{2}(Q)^{N}} \\
& \leq C\left\|\mathrm{e}^{13 / 4 s \beta^{*}}\left(\gamma^{*}\right)^{-1-1 / m} w^{1}\right\|_{L^{2}\left(0, T ; L^{\infty}(\Omega)^{N}\right)}\left\|\mathrm{e}^{13 / 4 s \beta^{*}}\left(\gamma^{*}\right)^{-1-1 / m} w^{2}\right\|_{L^{\infty}(0, T ; V)},
\end{aligned}
$$

and the continuity follows since $25 / 4<13 / 2$ and thanks to $(4.3)$. The terms $\left(z \cdot \nabla^{t}\right) w,(w \cdot \nabla) z$ are treated analogously.

Finally, we can prove in the same way that the bilinear operator

$$
\left(\left(w^{1}, p_{0}^{1}, z^{1}, p_{1}^{1}, r^{1}, q^{1}, v^{1}, v_{0}^{1}\right),\left(w^{2}, p_{0}^{2}, z^{2}, p_{1}^{2}, r^{2}, q^{2}, v^{2}, v_{0}^{2}\right)\right) \rightarrow\left(w^{1} \cdot \nabla r^{2}, w^{1} \cdot \nabla q^{2}\right)
$$

is continuous from $\mathcal{G}_{1} \times \mathcal{G}_{1}$ to $L^{2}\left(\mathrm{e}^{25 / 4 s \beta^{*}}(0, T) ; L^{2}(\Omega)^{2}\right)$ just by taking into account that

$$
\mathrm{e}^{13 / 4 s \beta^{*}}\left(\left(\gamma^{*}\right)^{-1-1 / m} r,\left(\gamma^{*}\right)^{-15-15 / m} q\right) \in L^{\infty}\left(0, T ; H_{0}^{1}(\Omega)^{2}\right),
$$


for any $\left(w, p_{0}, z, p_{1}, r, q, v, v_{0}\right) \in \mathcal{G}_{1}$.

It is readily seen that $\mathcal{F}^{\prime}(0): \mathcal{G}_{1} \rightarrow \mathcal{G}_{2}$ is given by

$$
\begin{aligned}
\mathcal{F}^{\prime}(0)\left(w, p_{0}, z, p_{1}, r, q, v, v_{0}\right)= & \left(\mathcal{L} w+\nabla p_{0}-v \mathbb{1}_{\omega}-r e_{N}, \mathcal{L}^{*} z+\nabla p_{1}-w \mathbb{1}_{\mathcal{O}},\right. \\
& \left.\mathcal{L} r-v_{0} \mathbb{1}_{\omega}, \mathcal{L}^{*} q-z_{N}-r \mathbb{1}_{\mathcal{O}}\right)
\end{aligned}
$$

for all $\left(w, p_{0}, z, p_{1}, r, s, v, v_{0}\right) \in \mathcal{G}_{1}$. It follows that this functional is surjective in view of the null controllability result for the linear system given by Proposition 4.4.

Now, we are in condition to apply Theorem 5.1. By taking $g_{1}=0$ and $g_{2}=0$, it gives the existence of $\delta>0$ such that, if $\left\|\mathrm{e}^{C / t^{m}}\left(f, f_{0}\right)\right\|_{L^{2}(Q)^{N+1}} \leq \delta$, for some $C>0$, then we can find $\left(w, p_{0}, z, p_{1}, r, q, v, v_{0}\right) \in \mathcal{G}_{1}$ solution of (5.1). In particular, $v_{i_{0}} \equiv v_{N} \equiv 0$ and $(z(0), q(0))=(0,0)$ (see Rem. 4.3). Therefore, the proof of Theorem 1.1 is complete.

Acknowledgements. The authors would like to thank the "Agence Nationale de la Recherche" (ANR), Project CISIFS, grant ANR-09-BLAN-0213-02, for partially supporting this work, and also the referees for their meticulous revision and useful comments that helped to improve this paper.

\section{REFERENCES}

[1] V.M. Alekseev, V.M. Tikhomirov and S.V. Fomin., Optimal Control, Translated from the Russian by V.M. Volosov, Contemporary Soviet Mathematics. Consultants Bureau, New York (1987).

[2] O. Bodart and C. Fabre, Controls insensitizing the norm of the solution of a semilinear heat equation. J. Math. Anal. Appl. 195 (1995) 658-683.

[3] N. Carreño, Local controllability of the $N$-dimensional Boussinesq system with $N-1$ scalar controls in an arbitrary control domain. Math. Control Relat. Fields 2 (2012) 361-382.

[4] N. Carreño and S. Guerrero, Local null controllability of the $N$-dimensional Navier-Stokes system with $N-1$ scalar controls in an arbitrary control domain. J. Math. Fluid Mech. 15 (2013) 139-153.

[5] N. Carreño and M. Gueye, Insensitizing controls with one vanishing component for the Navier-Stokes system. J. Math. Pures Appl. 101 (2014) 27-53.

[6] J.-M. Coron and S. Guerrero, Null controllability of the $N$-dimensional Stokes system with $N-1$ scalar controls. J. Differ. Equ. 246 (2009) 2908-2921.

[7] J.-M. Coron and P. Lissy, Local null controllability of the three-dimensional Navier-Stokes system with a distributed control having two vanishing components. To appear in Invent. Math.

[8] J.I. Díaz and A.V. Fursikov, Approximate controllability of the Stokes system on cylinders by external unidirectional forces. J. Math. Pures Appl. 9 (1997) 353-375.

[9] E. Fernández-Cara, S. Guerrero, O. Yu. Imanuvilov and J.-P. Puel, Some controllability results for the N-dimensional Navier-Stokes and Boussinesq systems with $N-1$ scalar controls. SIAM J. Control Optim. 45 (2006) $146-173$.

[10] A.V. Fursikov O. Yu. Imanuvilov. Controllability of Evolution Equations. Lecture Notes \#34. Seoul National University, Korea (1996).

[11] S. Guerrero, Controllability of systems of Stokes equations with one control force: existence of insensitizing controls. Ann. Inst. Henri Poincaré Anal. Non Linéaire 24 (2007) 1029-1054.

[12] M. Gueye, Insensitizing controls for the Navier-Stokes equations. Ann. Inst. Henri Poincaré Anal. Non Linéaire 30 (2013) $825-844$.

[13] O. Yu. Imanuvilov, Remarks on exact controllability for the Navier-Stokes equation. ESAIM: COCV 6 (2001) 39-37.

[14] O. Yu. Imanuvilov, J.-P. Puel and M. Yamamoto, Carleman estimates for parabolic equations with nonhomogeneous boundary conditions. Chin. Ann. Math. Ser. B 30 (2009) 333-378.

[15] O. Kavian and L. de Teresa, Unique continuation principle for systems of parabolic equations. ESAIM: COCV 16 (2010) $247-274$.

[16] O.A. Ladyzhenskaya, The mathematical theory of viscous incompressible flow, revised English edition, translated from the Russian by Richard A. Silverman. Gordon and Breach Science Publishers, New York, London (1963).

[17] O.A. Ladyženskaja, V.A. Solonnikov and N.N. Ural'ceva, Linear and quasilinear equations of parabolic type, Translated from the Russian by S. Smith. Vol. 23 of Translations of Mathematical Monographs. American Mathematical Society, Providence, R.I. (1968).

[18] J.-L. Lions, Quelques notions dans l'analyse et le contrôle de systèmes à données incomplètes (Somes notions in the analysis and control of systems with incomplete data). Proc. of the XIth congress on Differential Equations and Applications/First Congress on Applied Mathematics, Málaga (1989). Univ. Málaga (1990) 43-54. 
[19] J.-L. Lions, Sentinelles pour les systèmes distribués à données incomplètes (Sentinelles for Distributed Systems with Incomplete Data). Vol. 21 of Recherches en Mathématiques Appliquées (Research in Applied Mathematics). Masson, Paris (1992).

[20] J.-L. Lions and E. Magenes, Problèmes aux limites non homogènes et applications, Vol. 2, Travaux et Recherches Mathématiques, No. 18. Dunod, Paris (1968).

[21] J.-L. Lions and E. Zuazua, A generic uniqueness result for the Stokes system and its control theoretical consequences, Partial differential equations and applications. Vol. 177 of Lect. Notes Pure Appl. Math. Dekker, New York (1996) $221-235$.

[22] S. Micu, J.H. Ortega and L. de Teresa, An example of $\varepsilon$-insensitizing controls for the heat equation with no intersecting observation and control regions. Appl. Math. Lett. 17 (2004) 927-932.

[23] R. Pérez-García, Nuevos resultados de control para algunos problemas parabólicos acoplados no lineales: controlabilidad y controles insensibilizantes. Ph.D. thesis, University of Seville, Spain, 2004.

[24] J.-C. Saut and B. Scheurer, Unique continuation for some evolution equations. J. Differ. Equ. 66 (1987) 118-139.

[25] R. Temam, Navier-Stokes Equations, Theory and Numerical Analysis. Vol. 2 of Study Math. Appl. North-Holland, Amsterdam, New York, Oxford (1977).

[26] L. de Teresa, Insensitizing controls for a semilinear heat equation. Commun. Partial Differ. Equ. 25 (2000) $39-72$. 